\title{
KBTBD13 is an actin-binding protein that modulates muscle kinetics
}

\author{
Josine M. de Winter, ${ }^{1}$ Joery P. Molenaar, ${ }^{2,3}$ Michaela Yuen, ${ }^{1,4}$ Robbert van der Pijl, ${ }^{1,5}$ Shengyi Shen, ${ }^{5}$ Stefan Conijn, ${ }^{1}$ \\ Martijn van de Locht, ${ }^{1}$ Menne Willigenburg, ${ }^{1}$ Sylvia J.P. Bogaards, ${ }^{1}$ Esmee S.B. van Kleef, ${ }^{2}$ Saskia Lassche, ${ }^{2}$ Malin Persson, ${ }^{6,7}$ \\ Dilson E. Rassier, ${ }^{6}$ Tamar E. Sztal, ${ }^{8}$ Avnika A. Ruparelia, ${ }^{8}$ Viola Oorschot, ${ }^{9}$ Georg Ramm, ${ }^{9,10}$ Thomas E. Hall, ${ }^{11}$ Zherui Xiong, ${ }^{11}$ \\ Christopher N. Johnson, ${ }^{12}$ Frank Li, ${ }^{5}$ Balazs Kiss, ${ }^{5}$ Noelia Lozano-Vidal, ${ }^{1}$ Reinier A. Boon, ${ }^{1}$ Manuela Marabita, ${ }^{13}$ Leonardo Nogara, ${ }^{13}$ \\ Bert Blaauw, ${ }^{13}$ Richard J. Rodenburg, ${ }^{14}$ Benno Küsters, ${ }^{15}$ Jonne Doorduin, ${ }^{2}$ Alan H. Beggs, ${ }^{16}$ Henk Granzier, ${ }^{5}$ Ken Campbell, ${ }^{17}$ \\ Weikang Ma, ${ }^{18}$ Thomas Irving, ${ }^{18}$ Edoardo Malfatti, ${ }^{19}$ Norma B. Romero, ${ }^{20,21}$ Robert J. Bryson-Richardson, ${ }^{7}$ \\ Baziel G.M. van Engelen, ${ }^{2}$ Nicol C. Voermans, ${ }^{2}$ and Coen A.C. Ottenheijm ${ }^{1,5}$
}

'Department of Physiology, Amsterdam University Medical Center, Netherlands. 'Department of Neurology, Donders Institute for Brain, Cognition and Behaviour, Radboud University Medical Center, Nijmegen, Netherlands. ${ }^{3}$ Department of Neurology, Rijnstate Hospital, Arnhem, Netherlands. ${ }^{4}$ Discipline of Paediatrics and Child Health, Faculty of Medicine, University of Sydney, Australia. ${ }^{5}$ Department of Cellular and Molecular Medicine, University of Arizona, Tucson, Arizona, USA. ${ }^{6}$ Department of Kinesiology and Physical Education, McGill University, Montreal, Canada. ${ }^{7}$ Department of Physiology and Pharmacology, Karolinska Institutet, Stockholm, Sweden. ${ }^{8}$ School of Biological Sciences, Monash University, Melbourne, Australia. ${ }^{9}$ Monash Ramaciotti Centre for Structural Cryo-Electron Microscopy, Monash University, Melbourne, Australia. ${ }^{10}$ Biochemistry and Molecular Biology, Monash Biomedicine Discovery Institute, Monash University, Melbourne, Victoria, Australia. "Institute for Molecular Bioscience, University of Queensland, Queensland, Australia. ${ }^{2}$ Division of Clinical Pharmacology, Center for Arrhythmia Research and Therapeutics and Center for Structural Biology, Vanderbilt University Medical Center, Nashville, Tennessee, USA. ${ }^{13}$ Venetian Institute of Molecular Medicine, Department of Biomedical Sciences, University of Padova, Italy. ${ }^{14}$ Department of Pediatrics, Radboud University Medical Centre, Translational Metabolic Laboratory, Nijmegen, Netherlands. ${ }^{15}$ Department of Pathology, Radboud University Medical Centre, Nijmegen, Netherlands. ${ }^{16}$ Division of Genetics and Genomics, The Manton Center for Orphan Disease Research, Boston Children's Hospital, Harvard Medical School, Boston, Massachusetts, USA. "Department of Physiology and Division of Cardiovascular Medicine, University of Kentucky, Lexington, Kentucky, USA. ${ }^{18}$ BioCAT, Illinois Institute of Technology, Chicago, Illinois, USA. ${ }^{19}$ Service Neurologie Médicale, Centre de Référence Maladies Neuromusculaire Paris-Nord CHU RaymondPoincaré, U1179 UVSQ-INSERM Handicap Neuromusculaire: Physiologie, Biothérapie et Pharmacologie Appliquées, UFR des Sciences de la Santé Simone Veil, Université Versailles-Saint-Quentinen-Yvelines, Garches, France. ${ }^{20}$ Sorbonne Université, Myology Institute, Neuromuscular Morphology Unit, Center for Research in Myology, GH Pitié-Salpêtrière Paris, France. ${ }^{21}$ Centre de Référence de Pathologie Neuromusculaire Paris-Est, CHU Pitié-Salpêtrière, Assistance Publique-Hôpitaux de Paris, Paris, France.

\begin{abstract}
The mechanisms that modulate the kinetics of muscle relaxation are critically important for muscle function. A prime example of the impact of impaired relaxation kinetics is nemaline myopathy caused by mutations in KBTBD13 (NEM6). In addition to weakness, NEM6 patients have slow muscle relaxation, compromising contractility and daily life activities. The role of КВTBD13 in muscle is unknown, and the pathomechanism underlying NEM6 is undetermined. A combination of transcranial magnetic stimulation-induced muscle relaxation, muscle fiber- and sarcomere-contractility assays, low-angle $x$-ray diffraction, and superresolution microscopy revealed that the impaired muscle-relaxation kinetics in NEM6 patients are caused by structural changes in the thin filament, a sarcomeric microstructure. Using homology modeling and binding and

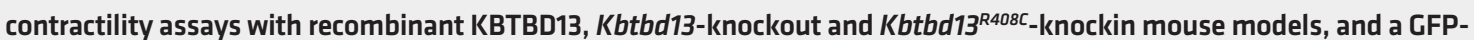
labeled Kbtbd13-transgenic zebrafish model, we discovered that KBTBD13 binds to actin - a major constituent of the thin filament - and that mutations in KBTBD13 cause structural changes impairing muscle-relaxation kinetics. We propose that this actin-based impaired relaxation is central to NEM6 pathology.
\end{abstract}

\section{Introduction}

Regulation of muscle contraction is an intricate interplay between the actin-based thin filament and the myosin-based thick filament, both key components of the sarcomere, the

\section{Related Commentary: p. 593}

Authorship note: JMDW, JPM, BCMVE, and NCV contributed equally to this work. Conflict of interest: The authors have declared that no conflict of interest exists. Copyright: ( 2020 , American Society for Clinical Investigation.

Submitted: August 16, 2018; Accepted: October 24, 2019; Published: January 6, 2020. Reference information: / Clin Invest. 2020;130(2):754-767.

https://doi.org/10.1172/JCI124000. smallest contractile unit in muscle (Figure 1A). During muscle activation, the number of force-generating myosin molecules (i.e., cross bridges) rapidly builds and high forces are generated; during relaxation, this number rapidly falls to bring force down and allow lengthening of passive muscle to occur. The kinetics of muscle activation have been widely studied, but relaxation is much less well understood, despite its crucial importance for muscle contractility and disease (1-3). A prime example of the impact of impaired relaxation kinetics on muscle contractility is nemaline myopathy (NEM), caused by mutations in KBTBD13 (NEM6). In addition to weakness, NEM6 patients have slow muscle relaxation (4-6), which impairs their ability to perform fast movements, e.g., running or climbing the stairs (see Supple- 
A

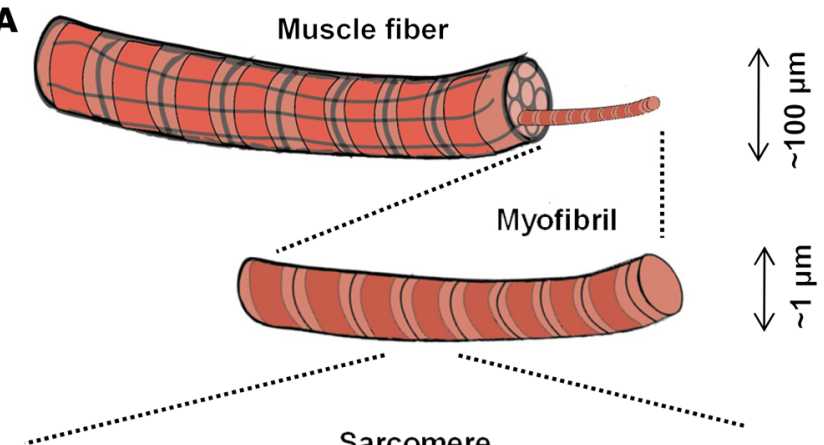

Sarcomere
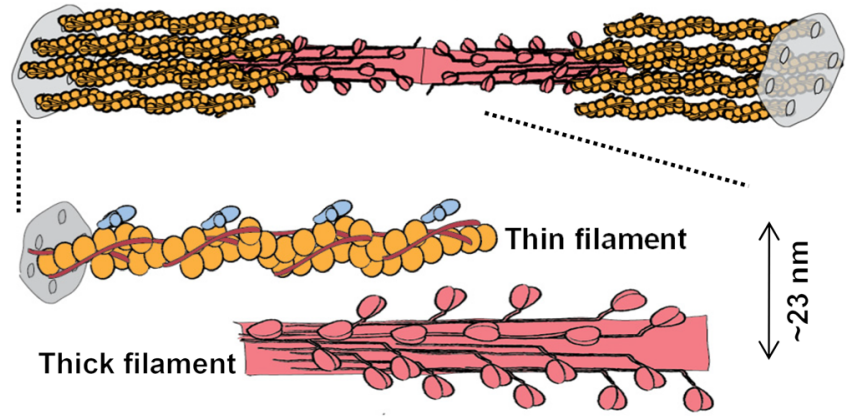

B

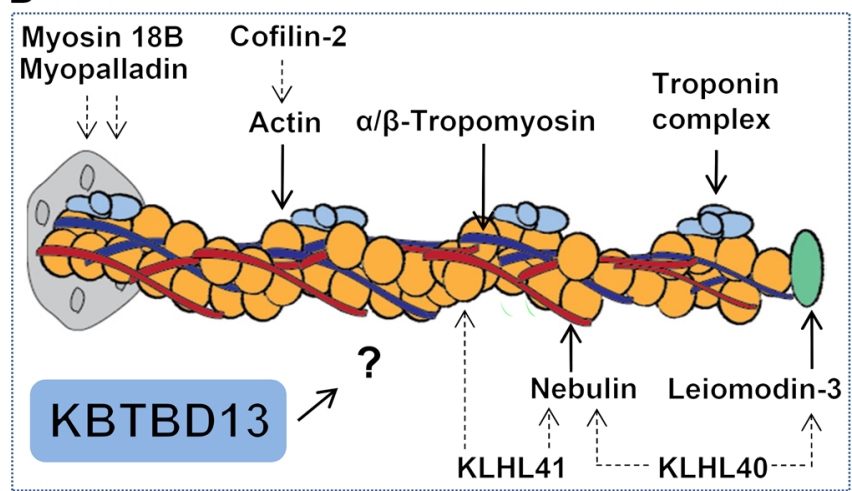

mental Videos 1-3; supplemental material available online with this article; https://doi.org/10.1172/JCI124000DS1), and to prevent themselves from falling when tripping.

NEMs are among the most common nondystrophic congenital myopathies (7). So far, 13 genes have been implicated: $\alpha$-actin 1 (ACTA1) (8), $\alpha$ - and $\beta$-tropomyosin (TPM3 and TPM2) (9, $10)$, nebulin (NEB) (11), leiomodin-3 (LMOD3) (12), troponin $\mathrm{T}$ (TNNT1) (13) and TNNT3 (14), cofilin 2 (CFL2) (15), unconventional myosin 18B (MYO18B) (16), myopalladin (MYPN) (17), Kelch family members 40 (KLHL4O) and -41 (KLHL41) $(18,19)$, and Kelch repeat and BTB (POZ) domain containing 13 (KBTBD13) (20). Eleven out of these thirteen genes encode proteins that are associated with the actin-based thin filament, and recent studies revealed that thin-filament dysfunction is a major contributor to muscle weakness in NEM (12, 21-23). Hence NEM is considered a disease of the thin filament.

Interestingly, KBTBD13 has no established association with the thin filament (Figure 1B). In fact, the localization and function of KBTBD13 are obscure (24), and therefore, the pathomechanism underlying weakness and impaired relaxation kinetics of muscle in NEM6 patients is unknown. The goal of this study was to discover
Figure 1. Schematic of muscle from the macroscopic to the nanoscopic level. (A) Simplified schematic of a skeletal muscle fiber, myofibril, sarcomere, and thick and thin filaments: key components of muscle activation and relaxation. (B) Simplified schematic of the skeletal muscle thin filament. The majority of genes implicated in NEM encode thin-filament (associated) proteins. For KBTBD13, the localization and function are unknown. Arrows indicate direct associations; dotted arrows indicate indirect associations.

this pathomechanism. As the vast majority of genes implicated in NEM encode thin-filament-associated proteins, we hypothesized that the impaired muscle-relaxation kinetics in NEM6 patients are caused by changes in thin-filament structure and function.

To test this hypothesis, we applied techniques to study muscle relaxation at various levels of organization, ranging from the patient in vivo to individual sarcomeres isolated from patient biopsies. A combination of transcranial magnetic stimulationinduced (TMS-induced) in vivo muscle-relaxation studies, in vitro muscle fiber- and sarcomere-contractility assays, and low-angle x-ray diffraction and superresolution microscopy revealed that the impaired muscle-relaxation kinetics in NEM6 patients is thin filament based. Using homology modeling, binding, and contractility assays with recombinant KBTBD13, Kbtbd13-KO

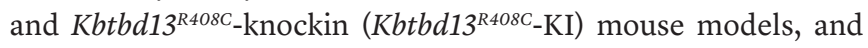
a GFP-labeled Kbtbd13-transgenic zebrafish model, we discovered that KBTBD13 is an actin-binding protein and that mutations in KBTBD13 affect the kinetics of muscle relaxation through effects on thin-filament structure. Hence, this study identifies (a) KBTBD13 as a regulator of skeletal muscle relaxation and (b) the pathomechanism underlying muscle dysfunction in NEM6 myopathy as thin-filament-based impaired muscle-relaxation kinetics.

\section{Results}

Lower muscle force and slower relaxation kinetics in NEM6 patients in vivo Patient characteristics are shown in Table 1. In vivo muscle force and relaxation kinetics of deep-finger flexors were determined using noninvasive TMS (25) (Figure 2A). Maximal force was lower in NEM6 patients than in control subjects, both in males and in females (Figure 2B). Because relaxation kinetics were similar between males and females, these data were pooled. Figure $2 \mathrm{~A}$ shows a typical relaxation trace of a patient and control subject with marked slower muscle relaxation. The normalized peak relaxation rate was lower in NEM6 patients than in controls (Figure $2 \mathrm{C}$ ), and accordingly, the $75 \%$ relaxation time (time for force to drop by 25\%) was longer in NEM6 patients than in controls (Figure 2D). This demonstrates that the origin of slower muscle relaxation in NEM6 patients is myogenic.

\section{Lower contractile force and slower relaxation kinetics in NEM6 muscle fibers}

First, we studied whether changes in $\mathrm{Ca}^{2+}$ handling proteins contributed to impaired muscle relaxation in NEM6. The level of SERCA1, a key protein involved in sarcoplasmic reticulum $\mathrm{Ca}^{2+}$ handling in fast-twitch fibers, was not significantly different between NEM6 and control muscle (Supplemental Figure 1, A and B). SERCA1 protein levels and SERCA activity strongly correlated with 
Table 1. Clinical characteristics and genetic information of patients and controls

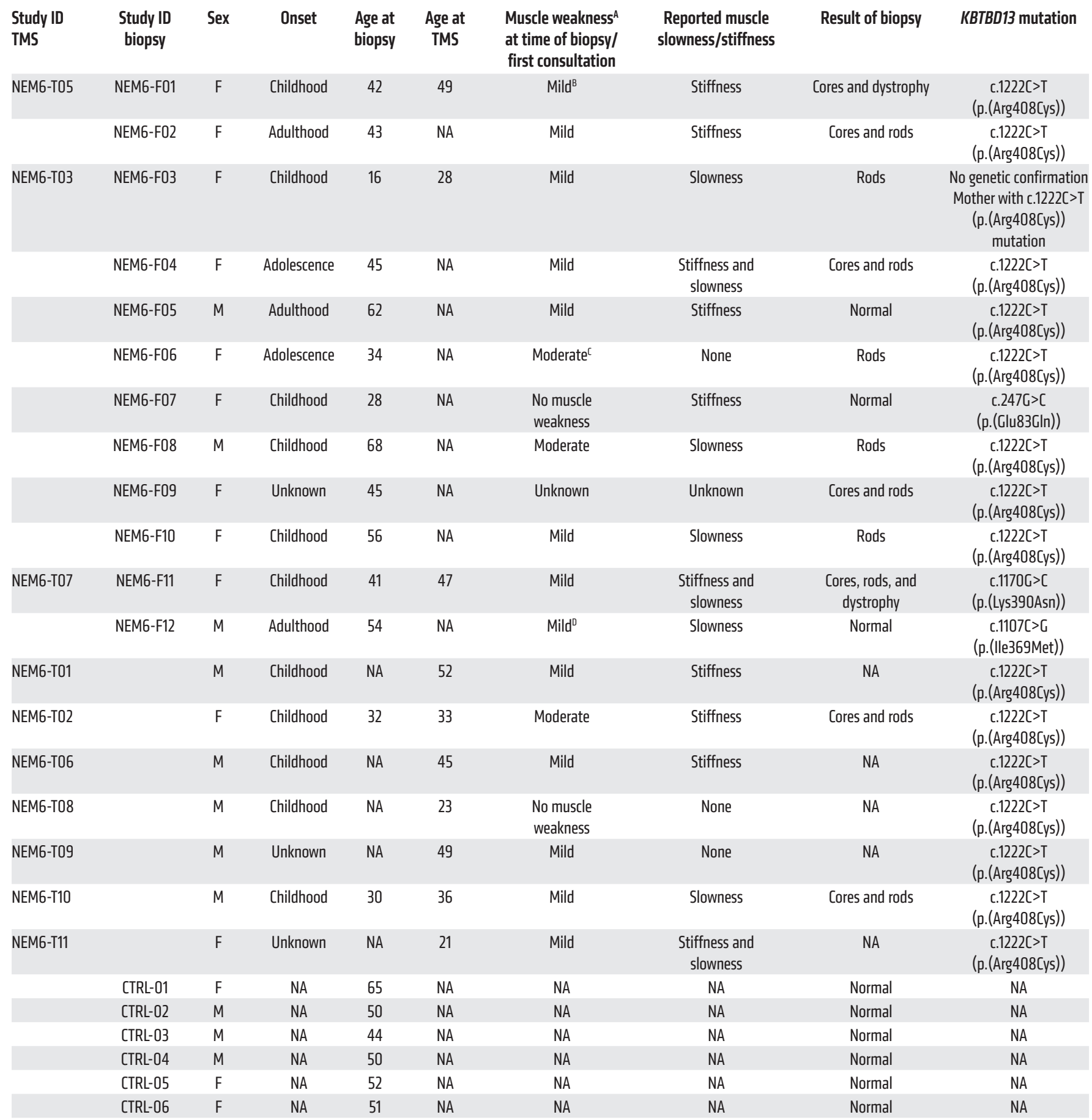

All the biopsies that showed cores and/or rods also showed myopathic changes, including an increase of internal nuclei, fiber type disproportion, and fiber type I predominance. NA, not assessed. AMuscle weakness: proximal muscle groups are more affected than distal ones. Axial weakness is also present. No facial weakness. ${ }^{B}$ Mild: strength is at least MRC scale 4. ${ }^{C}$ Moderate: strength is MRC scale $3-4 .{ }^{D}$ This patient has predominantly distal weakness and thus differs from the other patients.

the area of fast-twitch fibers in muscle biopsies of NEM6 patients (Supplemental Figure 1, C-E). The ratio of phospholamban (active form) over phosphorylated phospholamban (inactive form) was increased in NEM6 muscle biopsies (Supplemental Figure 1F).
As phospholamban slows SERCA activity, this might contribute to slower muscle relaxation in NEM6. Electron microscopy (EM) analyses showed no aberrations in the structure of the triads in muscle fibers of NEM6 patients. 
A
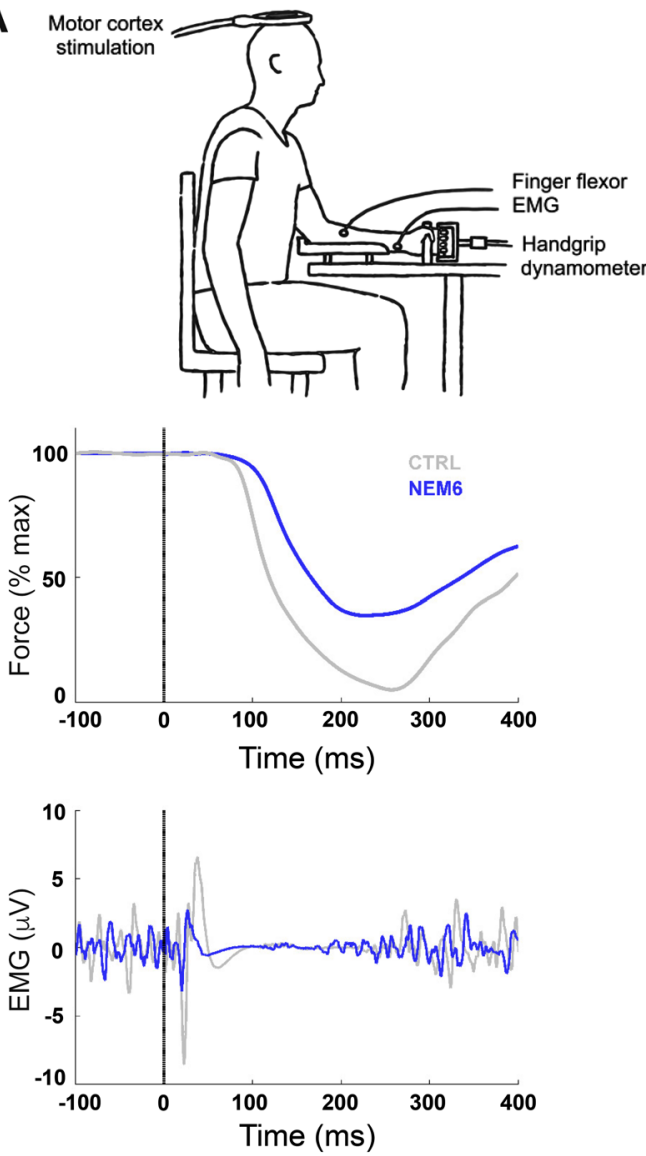

B

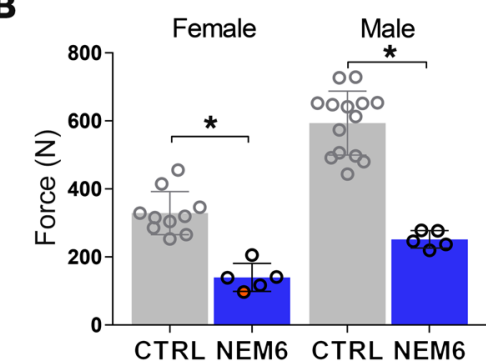

C

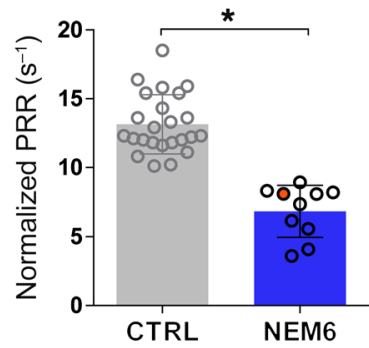

D

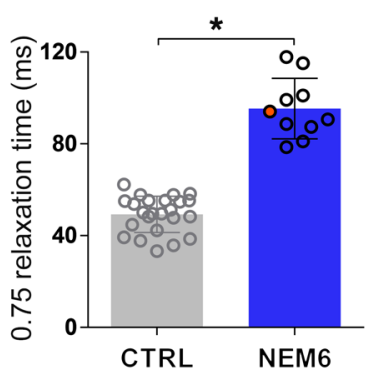

Figure 2. TMS to study in vivo muscle-relaxation kinetics. (A) Schematic of the experimental setup for TMS to induce involuntary muscle relaxation (top). Typical, superimposed, force traces, and corresponding electromyogram (EMG) of a control subject (CTRL) and NEMG patient during maximal voluntary contraction and TMS-induced involuntary relaxation of the deep-finger flexors (middle and bottom). Note the motor-evoked potential on the EMC traces corresponding to the small superimposed twitch and the EMC silent period of approximately $200 \mathrm{~ms}$ corresponding to the drop in force, after which voluntary force is generated again. Note the slower relaxation in the NEM6 patient. (B) Maximal force is lower in NEM6 patients than in controls, both in males and in females. (c) The peak relaxation rate (normalized to maximal force; pRR) is lower in NEM6 patients than in controls. (D) The time to $75 \%$ of maximal force ( 0.75 relaxation time) is longer in NEM6 patients than in controls. Orange circle corresponds to the NEM6 patient harboring the KBTBD13 ${ }^{\text {K390N }}$ mutation, white circles to those with the KBTBD13 ${ }^{\text {R408C }}$ mutation. Student's $t$ tests were performed between NEM6 and controls. ${ }^{*} P<$ 0.05 . For detailed information on the number of samples and statistical tests and outcomes, please see Supplemental Table 1.
Next, to study whether sarcomeric changes contribute to slower muscle-relaxation kinetics, we isolated permeabilized single-muscle fibers from biopsies of NEM6 patients and activated these with exogenous $\mathrm{Ca}^{2+}$. Patient characteristics are shown in Table 1. Figure 3, A and B, shows EM images of muscle fibers from a control and an NEM6 patient, and Figure 3, C and D, show images of representative slow-twitch and fast-twitch NEM6 patient fibers that were used for mechanics. In NEM6 patients, the ratio of slow-twitch/fast-twitch fiber cross-sectional area was increased (Supplemental Figure 2A), indicating that the fibers used for mechanics reflect the fiber size distribution in the whole biopsy as determined by histology (Supplemental Figure 2, B and C). Next, fibers were exposed to incremental $\mathrm{Ca}^{2+}$ concentrations and the resulting forces were recorded. Maximal absolute force (i.e., force at pCa 4.5) was lower in fast-twitch fibers of NEM6 patients compared with those of control subjects, but not in slow-twitch NEM6 fibers compared with those of controls (Supplemental Figure 2, D and $\mathrm{E}$ ). To correct for differences in fiber size, force was normalized to the cross-sectional area of the fiber (i.e., tension). In NEM6 patients, maximal tension of both slow-twitch and fast-twitch fibers was significantly lower than in fibers of control subjects (tension reduced by approximately 50\%; Figure 3, E and F). The lower maximal active tension was not caused by shorter thin-filament lengths: the sarcomere-length dependence of maximal tension was not different between NEM6 and control fibers (Supplemental Figure 2F). This finding was in line with the unaltered thin-filament length in NEM6 fibers, as determined by superreso- lution-stimulated emission depletion (STED) microscopy (Supplemental Figure 2F). The calcium sensitivity of both slow-twitch and fast-twitch fibers was increased in NEM6 patients, as reflected by the higher $\mathrm{pCa}_{50}$ (Supplemental Figure 2, D and E). Note that we observed no changes in the passive stiffness of NEM6 muscle fibers (Supplemental Figure 3), suggesting that titin does not contribute to the muscle stiffness experienced by NEM6 patients.

Importantly, the kinetics of relaxation after maximal activation were markedly slower in both slow-twitch and fast-twitch fibers of NEM6 patients when compared with control subjects. Figure $3 \mathrm{G}$ shows a typical relaxation trace of a patient's and a control subject's muscle fibers. The relaxation kinetics, reflected by the relaxation constant $K_{\text {rel }}$, were approximately 3-fold slower in NEM6 fibers (Figure 3H). Thus, our findings on permeabilized NEM6 muscle fibers suggest that the mechanism contributing to slower muscle-relaxation kinetics in NEM6 patients is at least partly sarcomere-based and independent of muscle-fiber type.

\section{Lower contractile force and slower relaxation kinetics in NEM6 myofibrils} Considering that a single fiber (diameter $\sim 100 \mu \mathrm{m}$ ) is a collection of myofibrils (diameter $\sim 1 \mu \mathrm{m}$; Figure $1 \mathrm{~A}$ ), contractile weakness of single fibers could be caused by ultrastructural damage or misalignment of myofibrils. Furthermore, the determination of relaxation kinetics in fibers could be confounded by the different size of the NEM6 fibers compared with control fibers (Supplemental Figure 2, A-C), which affects diffusion time of solutions. Therefore, we also measured the contractility of 
A

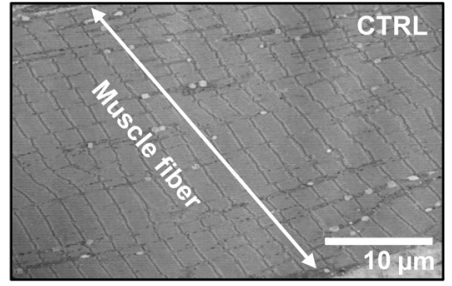

C

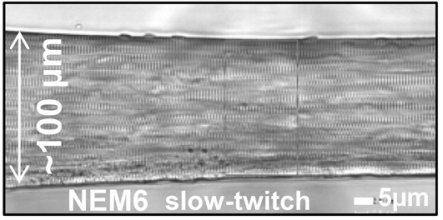

E

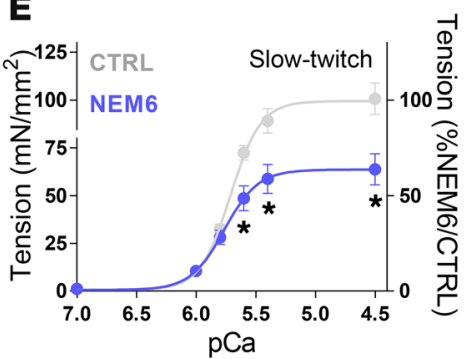

G

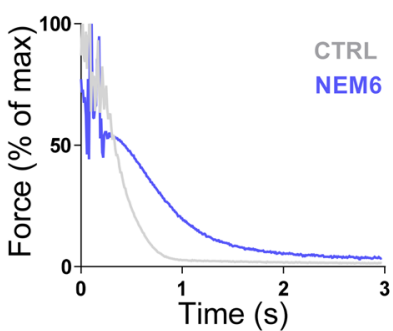

B

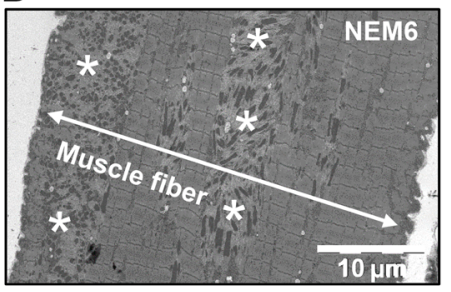

D

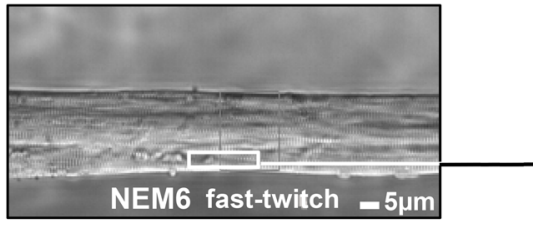

$\mathbf{F}$

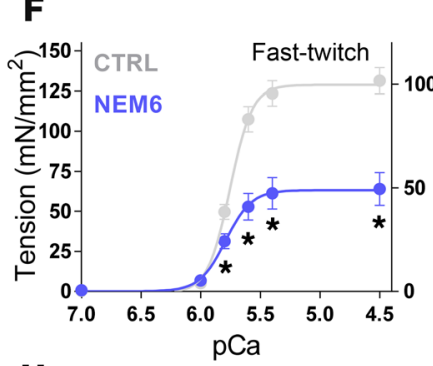

H

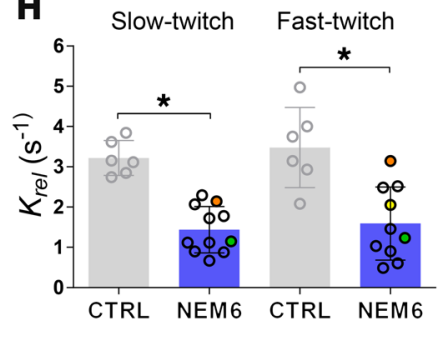

I

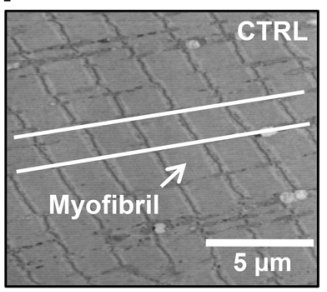

$\mathbf{K}$

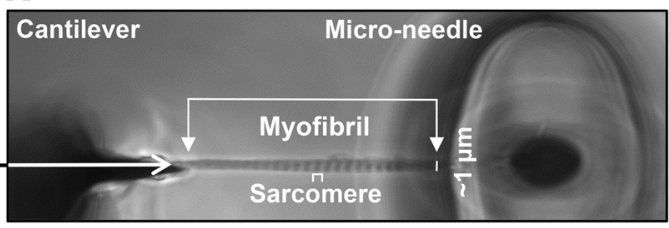

L

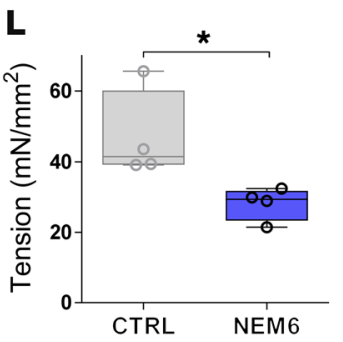

N

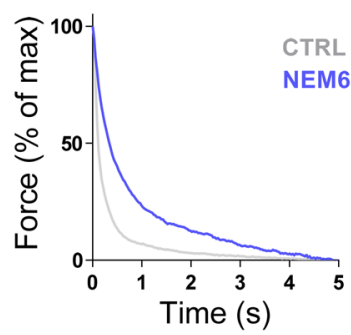

J

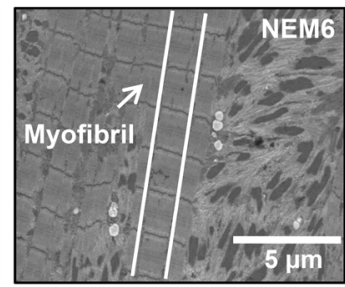

M

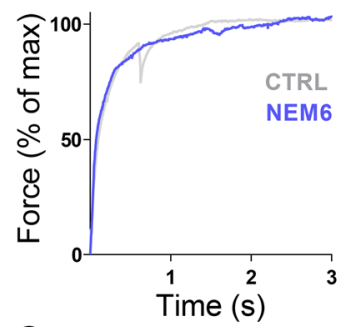

0

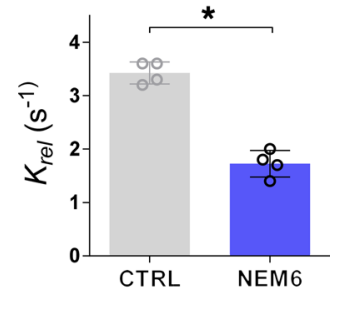

Figure 3. Contractility assays to study relaxation kinetics of muscle fibers and myofibrils. (A) EM images from muscle fibers of a control and (B) a NEM6 patient (note the areas with myofibrillar damage, indicated by asterisks). (C) Light microscopy images of a slow-twitch and (D) a fast-twitch NEM6 patient fiber that were used for contractility assays. (E) Maximal tension of both slow-twitch and (F) fast-twitch fibers from NEM6 patients was significantly lower than that of controls. (C) Typical relaxation trace of a NEM6 and a control muscle fiber. (H) The kinetics of relaxation after maximal activation - reflected by the relaxation constant $K_{\text {rel }}$ - were markedly slower in both slow-twitch and fast-twitch fibers of NEM6 patients when compared with controls. The

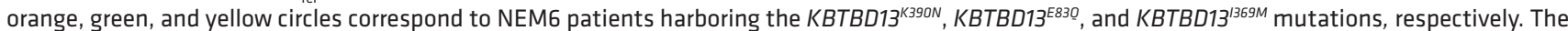
white circles correspond to patients with the $K B T B D 13^{R 408 C}$ mutation. (I) EM images indicate a myofibril (highlighted in white) from muscle fibers of control and (J) a NEM6 patient. Note that these EM images are higher magnifications of the same images in parts A and B, respectively. (K) Myofibril mounted between a force probe and length controller for contractility assays. (L) The maximal tension of myofibrils of NEM6 patients was lower than that of controls. (M) Typical example of the activating kinetics and (N) the relaxation kinetics of a NEM6 and a control myofibril; note that activation and relaxation kinetics are from the same myofibril. (0) The relaxation kinetics were slower in myofibrils of NEM6 patients than in those of controls. Student's $t$ tests or Mann-Whitney $U$ tests were performed between NEM6 and controls. ${ }^{*} P<0.05$. For each biopsy, 4 to 12 muscle fibers/myofibrils were measured. For detailed information on the number of samples and statistical tests and outcomes, please see Supplemental Table 1.

NEM6 myofibrils. Figure 3, A B, I, and J, shows EM images of a control and an NEM6 muscle fiber (1 myofibril is highlighted). The EM image of the NEM6 muscle fiber reveals that areas with myofibrillar damage as well as areas with preserved myofibrillar ultrastructure are present within 1 muscle fiber (note that nemaline rods were frequently observed in EM images of the NEM6 patients; for a high-magnification example, see Supplemental Figure 4). We isolated single myofibrils from the intact areas of NEM6 muscle fibers and measured their contractile function (experimental arrangement shown in Figure 3K). As shown in Figure 3L, the maximal tension of myofibrils of NEM6 patients was lower than that of control subjects. Thus, in addition to myofibrillar damage, myofibrillar dysfunction contributes to fiber weakness in NEM6 patients. Importantly, whereas the activating kinetics were on average similar between NEM6 and control myofibrils (an example is shown in Figure 3M), the relaxation kinetics were slower in myofibrils of NEM6 patients than in those of control subjects (Figure 3, N and O; note that Figure $3 \mathrm{~N}$ shows the same myofibril as in Figure $3 \mathrm{M}$ ). Thus, our findings on individual myofibrils indicate that slower sarcomeric relaxation kinetics contribute to slow muscle-relaxation kinetics in NEM6 patients. 
A

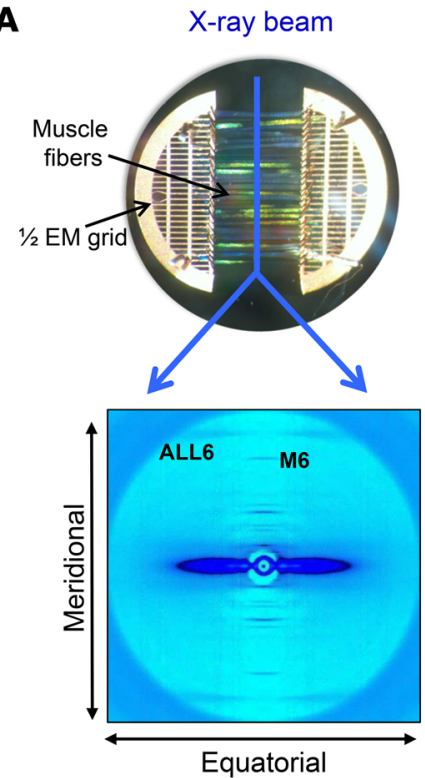

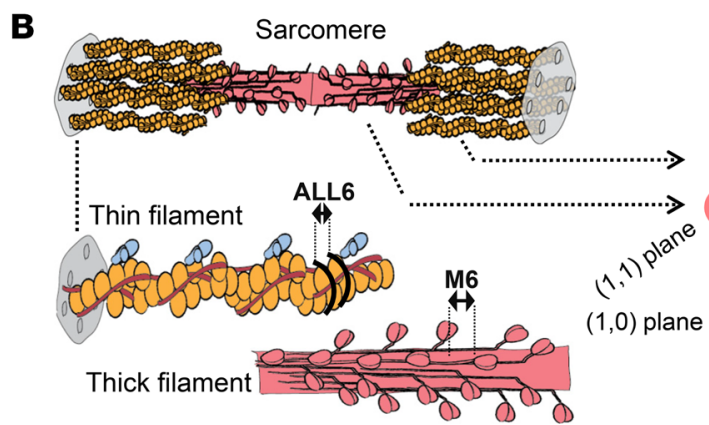

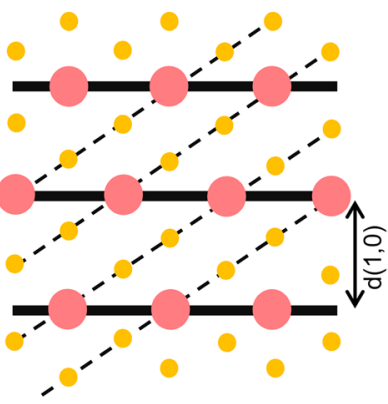

D

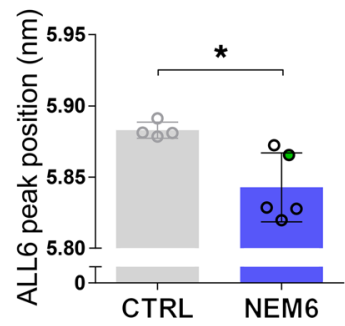

Figure 4. Determination of the nanostructure of muscle fibers. (A, top) Image of 28 NEM6 muscle fibers mounted and aligned in 1 plane between 2 halves of an EM grid (bottom); typical example of the resulting low-angle x-ray diffraction pattern. Note the well-resolved equatorial and meridional reflections. (B, left) Schematic of a sarcomere and the thin and thick filament, highlighting the structures that underlie the ALL6 reflection and the myosin (M6) reflection; (right) schematic representation of a cross section of muscle (thin filament in yellow, thick filament in pink), indicating the 1,0 and the 1,1 reflections. (C) No change in M6 reflection (thick-filament backbone periodicity) is observed in fibers of NEM6 patients compared with those of controls. (D) The ALL6 reflection is reduced in NEM6 fibers compared with control fibers. The green circles and white circles correspond to NEM6 patients harboring the KBTBD13 ${ }^{E 830}$ mutation and the KBTBD13 ${ }^{R 08 C}$ mutation, respectively. Student's $t$ tests or Mann-Whitney $U$ tests were performed between NEM6 and controls. ${ }^{*} P<0.05$. For detailed information on the number of samples and statistical tests and outcomes, please see Supplemental Table 1.

Structural changes in the thin filament suggest a stiffer thin filament in NEM6 patients.

As the findings from the fiber and myofibril studies point toward a sarcomere-based mechanism, we next determined whether structural changes in the thick and/or thin filament cause the slow-relaxation kinetics. At the Advanced Photon Source (Argonne National Laboratories), low-angle x-ray diffraction patterns were obtained from preparations in which 28 fibers were mounted and aligned in 1 plane between 2 halves of EM grids. This approach allowed for high-quality equatorial and meridional diffraction patterns (for example, see Figure $4 \mathrm{~A}$ ). The spacing of the equatorial reflections indicated that lattice spacing was not different between NEM6 and control fibers (distance between thick filament planes $\left(d_{1,0}\right)$; see Figure $4 B ; 40.76 \pm 0.37$ vs. $40.95 \pm 0.45 \mathrm{~nm}$, respectively), and the equatorial intensity ratios indicated that the position of myosin heads between the thick and thin filaments was not different between NEM6 and control fibers $\left(\mathrm{I}_{1,1} / \mathrm{I}_{1,0}\right.$, where $\mathrm{I}_{1,1}$ indicates intensity resulting from 1,1 planes; see Figure 4B); 0.71 \pm 0.13 vs. $0.68 \pm 0.05$, respectively). The meridional reflections indicated no spacing changes in thick-filament structure: both the myosin head displacement from the thick-filament backbone (M3 reflection: $14.53 \pm 0.02 \mathrm{~nm}$ for control and $14.46 \pm 0.03 \mathrm{~nm}$ for NEM6 fibers) and the thick-filament backbone periodicity (M6 reflection, Figure 4C) were comparable in fibers of NEM6 patients and those of controls. Reflections from the thin filament, however, were different between NEM6 and control fibers: the actin layer line 6 (ALL6) indicating the left-handed pitch of the thin-filament helix was shorter in NEM6 than in control fibers (Figure 4D).
Thus, low-angle x-ray diffraction studies suggest that the actinbased thin filament is more tightly wound in NEM6 muscle fibers, a change that might reflect increased thin-filament stiffness.

\section{Slower relaxation kinetics and stiffer thin filaments in muscle of Kbtbd13 ${ }^{R 408 C}$ mice}

To further investigate the effect of the Dutch founder mutation $K B T B D 13^{R 408 C}$ on muscle-relaxation kinetics and thin-filament stiffness, we engineered a Kbtbd13 ${ }^{R 408 C}$-KI mouse model (Figure 5A). Mice were studied at 9 months after birth (this age was chosen, as in patients the phenotype is slowly progressive).

Basic characteristics of the model. Homozygous mice were born at significantly higher than Mendelian ratio (Supplemental Figure 5A). Quantitative PCR showed that homozygous KI mice expressed only mutant $K b t b d 13$ and that heterozygous mice expressed approximately $50 \%$ of mutant and $50 \%$ of WT Kbtbd13 (Supplemental Figure 5C). KI mice had higher body weight than WT mice (Figure 5B). Soleus and diaphragm muscle weights were higher in KI compared with WT mice (Supplemental Figure 5D). Picrosirius red-stained cryosections of $\mathrm{m}$. soleus (representative images in Supplemental Figure 5E) showed a significantly increased area of extracellular matrix in KI compared with WT mice $(14 \% \pm 1 \%$ vs. $12 \% \pm 1 \%$, respectively). Soleus muscle of KI mice had a lower percentage of slow-twitch (type 1) fibers than that of WT mice $(28 \%$ $\pm 2 \%$ vs. $60 \% \pm 3 \%$, respectively; Supplemental Figure $5 \mathrm{E}$ ). The cross-sectional area of slow-twitch and fast-twitch (type 2A) fibers was larger in $\mathrm{m}$. soleus of KI than of WT mice (Supplemental Figure 5F). Similarly to what occurs in NEM6 patients (Supplemental Fig- 
A

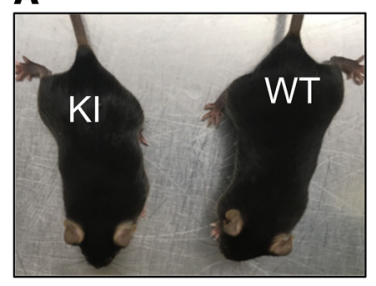

B

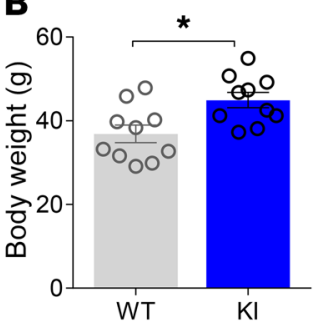

C

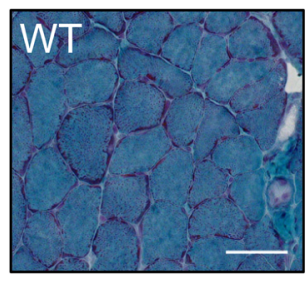

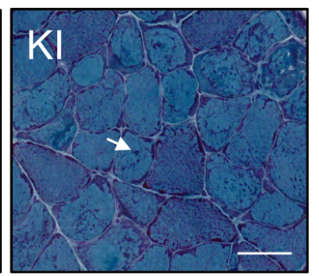

D

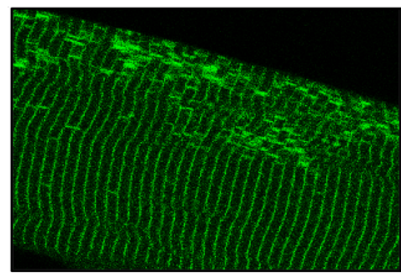

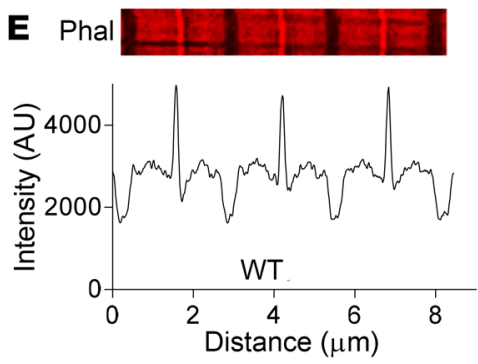
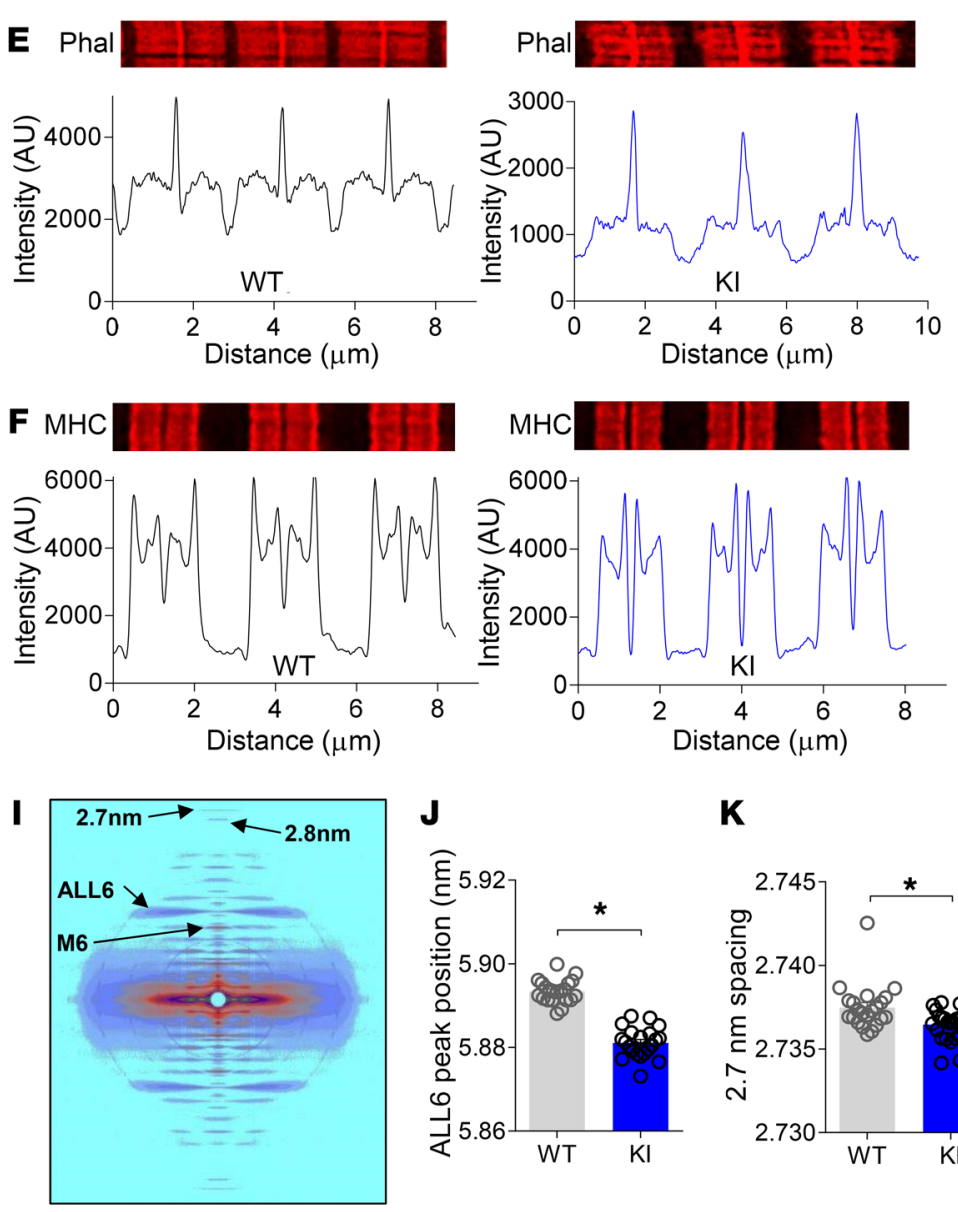

$\mathbf{K}$

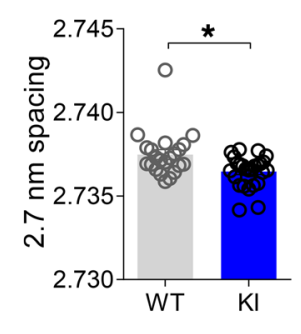

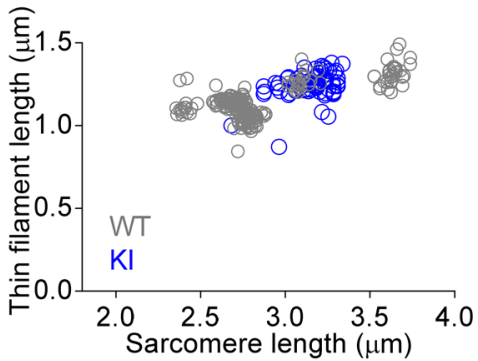

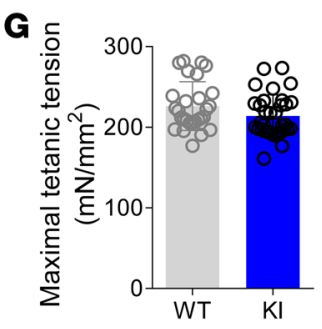

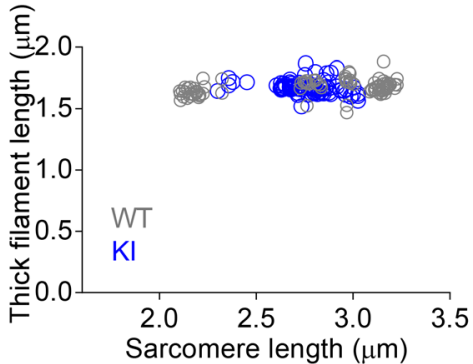

H

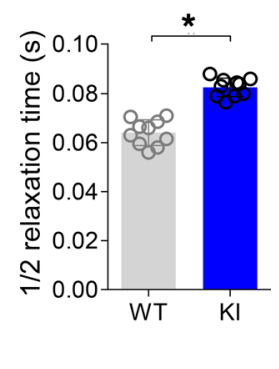

$\mathbf{L}$

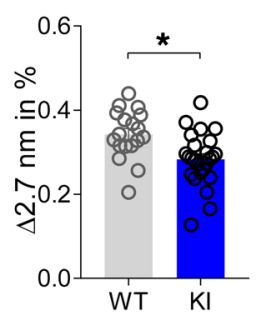

M

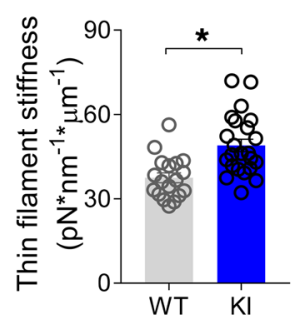

Figure 5. Characteristics of the Kbtbd13 ${ }^{R 408 C} \mathbf{K I}$ mouse model. (A) Image of Kbtbd13 ${ }^{R 408 C}$ WT and homozygous KI mouse. (B) Body weight was significantly higher in $\mathrm{KI}$ mice. (C) Gomöri trichrome staining of m. soleus cryosections. Scale bars: $100 \mu \mathrm{m}$. KI section shows nemaline rods in many muscle fibers (examples indicated by white arrow). (D) Phalloidin staining of a permeabilized fiber of m. soleus; note the abundance of actin-positive nemaline rods. (E and F) STED microscopy images (deconvolved) of permeabilized fibers of $\mathrm{m}$. soleus stained with phalloidin (Phal) (to stain the thin filament) and with a pan-specific myosin heavy chain (MHC) antibody (to stain the think filament); right panels show the resulting thin and thick-filament lengths as a function of sarcomere length. (G) Maximal tetanic tension $(150 \mathrm{~Hz}$ ) of $\mathrm{m}$. soleus was comparable between WT and KI mice. (H) The maximal tetanic half-relaxation time was significantly longer in m. soleus of KI than in WT mice. (I) Typical example of a low-angle X-ray diffraction pattern obtained from m. soleus of a Kbtbd13-KI mouse; note the well-resolved reflection of the actin subunit spacing $(2.7 \mathrm{~nm})$. (J) The spacing of the ALL6 reflection is reduced in muscle of $K b t b d 13^{R 408 C}-K I$ mice. (K) The actin subunit spacing $(2.7 \mathrm{~nm})$ is reduced in muscle of $K b t b d 13^{R 408 C}-\mathrm{KI}$ mice. $(\mathrm{L})$ The change in the actin subunit spacing $(\Delta 2.7 \mathrm{~nm})$ during maximal tetanic activation is smaller in $K_{b t b d 13^{R 408 C}-K I}$ mice, indicating less thin-filament extension. (M) Thin-filament stiffness is higher in muscle of $K b t b d 13^{R 408 C}-K I$ mice than in muscle of WT mice. Student's $t$ tests were performed between KI and WT mice. ${ }^{*} P<0.05$. For detailed information on the number of samples and statistical tests and outcomes, see Supplemental Table 1.

ure 2C), in KI mice, the cross-sectional area of slow-twitch fibers was larger than that of fast-twitch fibers (Supplemental Figure 5G). Gömöri trichrome-stained sections of $\mathrm{m}$. soleus showed nemaline bodies in muscle fibers (Figure 5C), a key characteristic of NEM. This finding was confirmed in fibers stained with phalloidin, showing actin-positive nemaline bodies (Figure 5D). Thin-filament and thick-filament length in muscle of KI mice was comparable to that in WT mice, as determined by superresolution STED microscopy (Figure 5, E and F). Thus, the pathology observed in Kbtbd13-KI mice resembled NEM6 pathology.

Relaxation kinetics. Maximal tetanic tension $(150 \mathrm{~Hz})$ of $\mathrm{m}$. soleus was comparable between KI and WT mice (Figure $5 \mathrm{G}$ ), 
and the force-frequency curve was shifted to the left (Supplemental Figure 5H). Importantly, similarly to observations in NEM6 patients, the relaxation kinetics of $\mathrm{m}$. soleus after maximal tetanic stimulation were slower in KI mice than in WT mice (Figure $5 \mathrm{H}$ ). After single-twitch stimulation, relaxation kinetics were also slower in KI mice compared with WT mice (Supplemental Figure 5I). The level of SERCA1 was not significantly different in KI compared with WT muscle (Supplemental Figure 5J).

Thin-filament stiffness. Next, we determined the structure of the thin and thick filaments in muscle of KI and WT mice. Low-angle $x$-ray diffraction patterns were obtained from intact $m$. soleus in the relaxed state and at maximal tetanic activation (Figure 5I). The meridional reflections indicated that, in the relaxed state, the structure of the thick filament was different in KI than in WT muscle: the thick-filament backbone periodicities (M6 reflection and the 2.8 $\mathrm{nm}$ reflection; Supplemental Figure 5, K and L) were both shorter in KI muscle than in WT muscle. However, the relative extension of the thick filament during maximal tetanic activation was comparable between KI and WT muscle ( $\Delta 2.8 \mathrm{~nm}$; Supplemental Figure $5 \mathrm{M})$, suggesting a comparable thick-filament stiffness.

Reflections from the thin filament were also different between KI and WT muscle. Similarly to the observations in NEM6 muscle, the ALL6 reflection was shorter in KI than in WT muscle (Figure 5J). Importantly, working with intact mouse muscles allowed us to determine actin subunit spacings (i.e., $2.7 \mathrm{~nm}$ reflections, Figure $5 \mathrm{~K})$. This reflection could not be resolved in the $\mathrm{x}$-ray diffraction patterns of permeabilized human muscle fibers (Figure 4A). We took advantage of this opportunity and observed that in relaxed muscle, the actin subunit spacing was shorter in KI than in WT mice (Figure 5K), supporting our notion that the shortened ALL6 reflection indicates a more compact thin filament. Then, to study whether the thin filament is stiffer in KI mice, we analyzed the change in the $2.7 \mathrm{~nm}$ reflection during maximal tetanic activation. As shown in Figure $5 \mathrm{~L}$, the change in this reflection $(\Delta 2.7 \mathrm{~nm})$ was reduced in KI muscle. Thus, this indicates that during activation, the thin filament extends less in KI muscle than in WT muscle. Next, we used the $\Delta 2.7 \mathrm{~nm}$ data to calculate the longitudinal stiffness of the thin filaments. To this end, it is important to investigate how the isometric tension is distributed over the myofilaments in the sarcomere. Hence, an ultrastructural analysis was performed on electron micrographs of KI and WT $\mathrm{m}$. soleus cross sections. No significant difference was observed in myofibrillar fractional area in KI compared with WT muscle (intermyofibrillar mitochondria: $3.6 \% \pm 0.1 \%$ vs. $2.4 \% \pm 0.4 \%$, respectively; subsarcolemmal mitochondria: $8.9 \% \pm 0.5 \%$ vs. $7.9 \% \pm 0.6 \%$, respectively). We then calculated the force per individual thin filament in activated muscle. Briefly, the unit cell area of the myofilament lattice was calculated from the $d_{1,0}$ equatorial spacing (in relaxed state: $34.2 \pm 0.1 \mathrm{vs}$. $32.7 \pm 0.2 \mathrm{~nm}, \mathrm{KI}$ vs. WT, respectively) according to Millman (26), with the number of unit cells per muscle cross section equaling the myofibril area (which was calculated using the mitochondrial area and the extracellular matrix area) divided by the unit cell area. Furthermore, the tension distribution along the thin and thick filaments was determined from their lengths (which were comparable between KI and WT muscle; Figure 5, E and F) and the overlap between them. For myofilament overlap, we assumed a sarcomere length of $2.8 \mu \mathrm{m}$ (based on Kiss and coworkers; ref. 27). The force obtained per thin filament was significantly higher in KI muscle than in WT muscle $(137.1 \pm 2.8 \mathrm{pN}$ vs. $124.6 \pm 2.1 \mathrm{pN}$, respectively). Normalizing this force to the extension of the thin filament $(\Delta 2.7$ $\mathrm{nm}$ reflection) showed that the force required to stretch the thin filament was significantly increased in KI muscle compared with WT muscle (Figure 5M). Thus, thin-filament stiffness is higher in muscle of KI mice.

\section{Normal relaxation kinetics in muscle of $K b t b d 13-K O$ mice}

We also generated a Kbtbd13-KO mouse model to study the effect of absence of Kbtbd13 on muscle-relaxation kinetics. Similarly to the mice of the Kbtbd13 ${ }^{\mathrm{R} 408 \mathrm{C}} \mathrm{KI}$ model, mice were studied 9 months after birth.

Basic characteristics of the model. Homozygous mice (KO; Supplemental Figure 6A) were born at close to Mendelian ratio (Supplemental Figure 6B). Gene Array Analyzer showed that Kbtbd13 mRNA levels were severely reduced in KO mice (Supplemental Figure 6C). KO mice had lower body weight than WT mice (Supplemental Figure 6D). Soleus muscle weights were lower in $\mathrm{KO}$ mice compared with WT mice (Supplemental Figure 6E), and quadriceps muscle weights were increased in $\mathrm{KO}$ mice.

Relaxation kinetics. The maximal tetanic tension $(150 \mathrm{~Hz})$ of $\mathrm{m}$. soleus was reduced in KO mice (Supplemental Figure 6F), and the force-frequency relation was comparable between KO and WT mice (Supplemental Figure 6G). The level of SERCA1 was not significantly different in KO compared with WT muscle (Supplemental Figure 6H). Importantly, the relaxation kinetics of both single twitches and maximal tetani were comparable between $\mathrm{KO}$ and WT mice (Supplemental Figure 6I). Thus, the relaxation kinetics of muscle of Kbtbd13-KO mice do not mimic those of Kbtbd13 ${ }^{\mathrm{R} 408 \mathrm{C}}-\mathrm{KI}$ mice and NEM6 patients.

\section{Relation between thin-filament stiffness and sarcomere relaxation kinetics}

To evaluate whether changes in thin-filament stiffness affect sarcomeric relaxation kinetics, simulations of paired compliant filaments were performed as described previously by Campbell (28) (for schematic, see Supplemental Figure 7A). Increasing the stiffness of the actin filaments slowed the rate of relaxation from $2.53 \mathrm{~s}^{-1}$ to $4.37 \mathrm{~s}^{-1}$ (Supplemental Figure 7B) without producing a substantial change in maximum isometric force (Supplemental Figure 7B). Thus, the combination of the results of the simulation experiments, the $\mathrm{x}$-ray diffraction experiments, and the contractility assays in patient biopsies and in mouse models suggest that mutations in KBTBD13 increase thin-filament stiffness and that increased thin-filament stiffness can slow sarcomeric relaxation kinetics.

\section{KBTBD13 binds to actin}

In order to examine the potential for KBTBD13 to bind to actin, we turned to homology modeling, anticipating that structural features of the protein provide insight into potential interactions. Given the low sequence similarity between KBTBD13 and other proteins $(<30 \%), 3$ independent programs with different template starting structures were used for the modeling process. We note a high degree of structural similarity between the structured regions of our 3 resulting models (root mean square deviation [R.M.S.D.] < $0.8 \AA$ ) that supports the relevance of our model. 
A
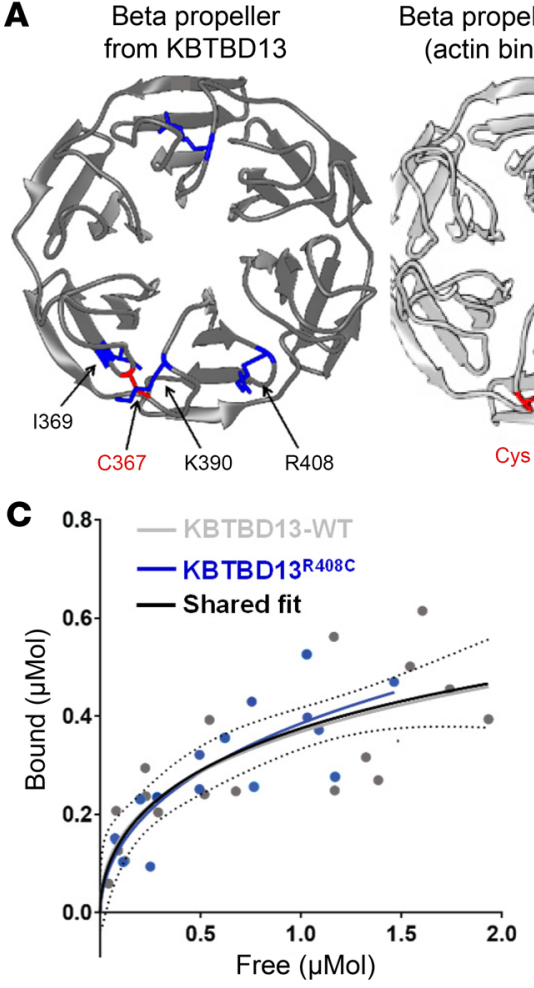

E
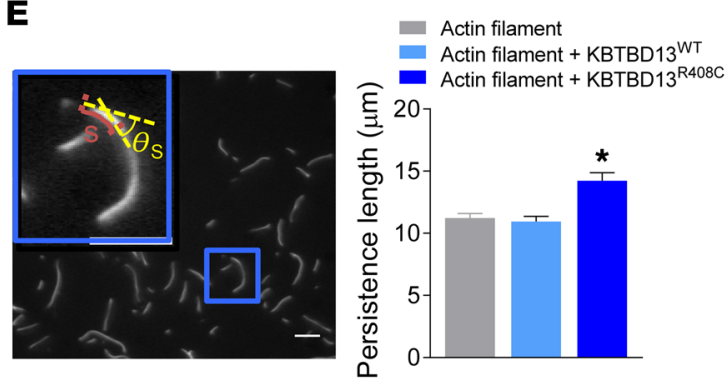

Beta propeller from scruin (actin binding protein)
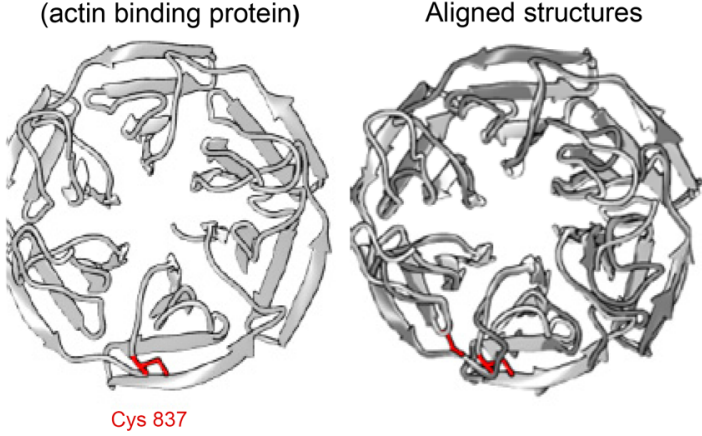

B

GST- $\quad$ GST-

+ KBTBD13 + GST- Protein muscle lys. muscle lys. KBTBD13 ladder

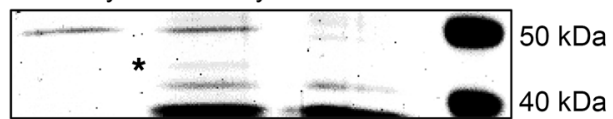

D

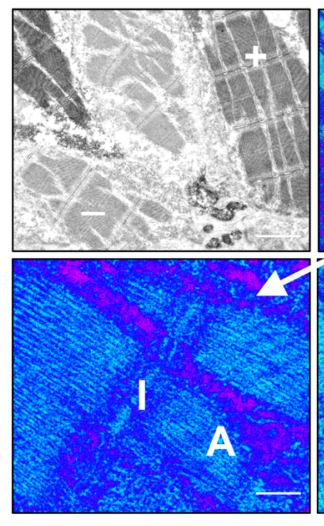

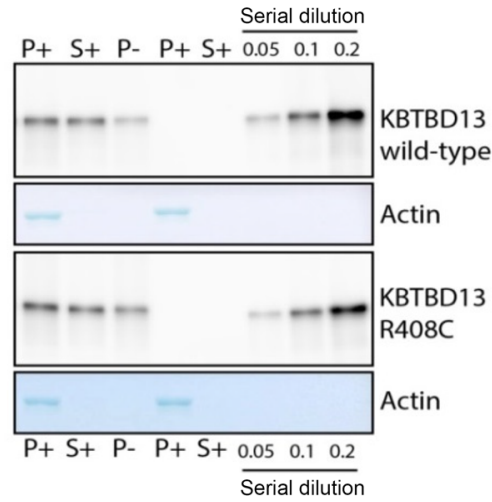
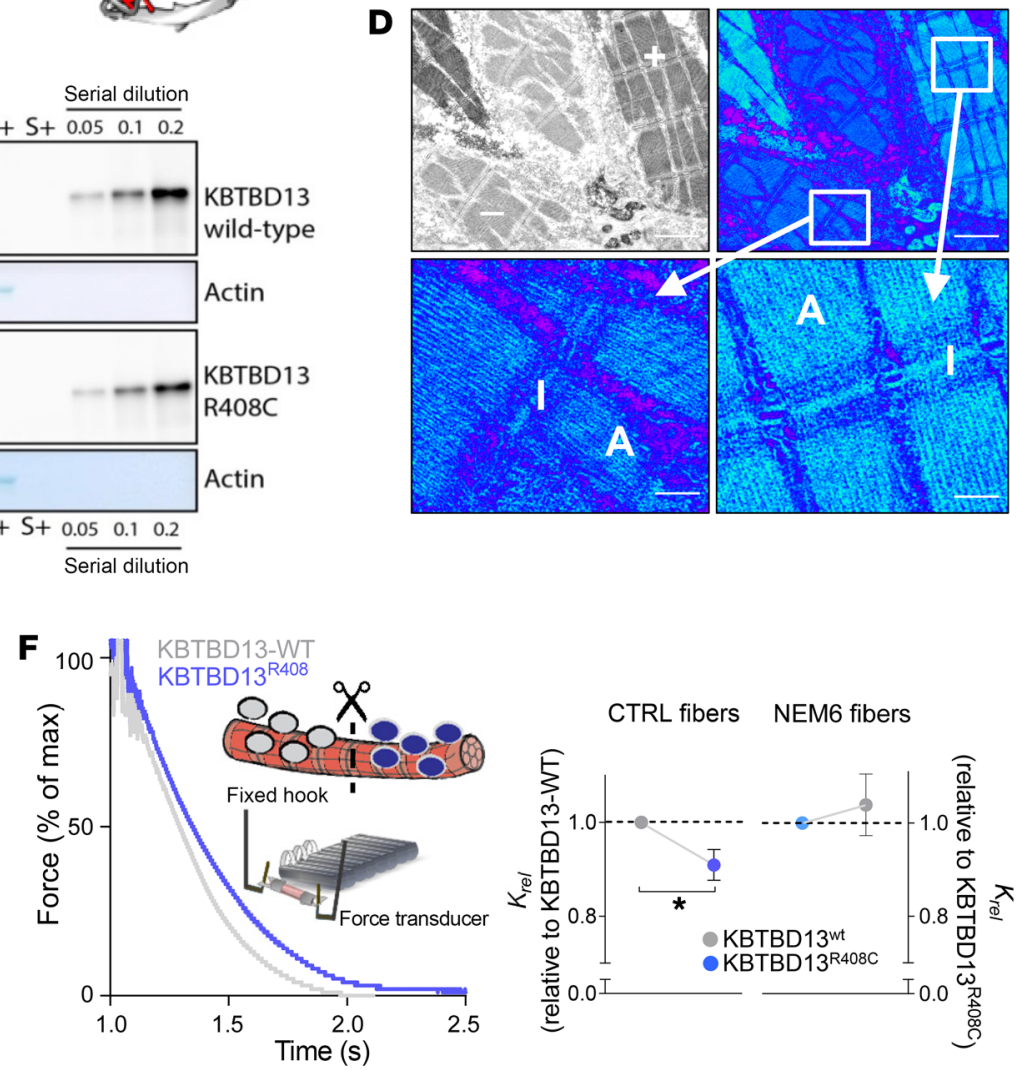

Figure 6. Effect of KBTBD13 on sarcomere structure and function. (A) Homology modeling reveals that KBTBD13 $\beta$ propeller motifs (left) have striking similarities with those of the actin-binding protein scruin in limulus sperm (middle). KBTBD13 has cysteine residue (Cys367) located in nearly the exact same position as scruin (Cys837, right). (B) SDS-PACE image after pulldown assay in a mouse muscle lysate (muscle lys.). One unique band (*) was identified, excised,

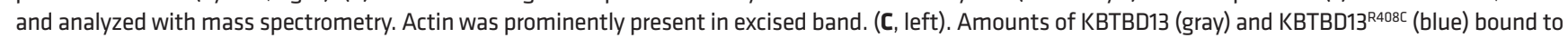
actin versus free KBTBD13 were plotted and fitted. The shared fit is shown in black. (C, right) Western blot of KBTBD13 in pellet and supernatant fraction (in presence of actin, $P_{+}$, and $S_{+}$, respectively) and pellet fraction in absence of actin ( $\left.P_{-}\right)$(lanes 1-3). Lanes 6-8 show dose-dependent detection of serial dilution of recombinant KBTBD13. (D) APEX-based EM of zebrafish expressing GFP-tagged KBTBD13. Positive cells stained dark in electron micrographs. Note that in cells, myofibrils in particular show dark staining. Scale bar: $1 \mu \mathrm{m}$. Top right: To increase contrast between KBTBD13-positive and -negative areas, images were converted from gray scale to spectrum scale, with KBTBD13 in light blue. Below, right: high magnification of myofibrillar compartment. Scale bar: $0.3 \mu \mathrm{m}$. Within myofibrils, A-band and I-band show labeling, indicating that KBTBD13 binds to thin filament and thick filament. Furthermore, z-discs, where thin filaments from adjacent sarcomeres overlap, show strong labeling. Below, left: high magnification of myofibrillar compartment of negative cell. Scale bar: $0.3 \mu \mathrm{m}$. (E) Left: representative image of actin filaments in solution. Inset illustrates determination of parameters required to calculate persistence length (Lp) of actin filaments. Right: KBTBD13 ${ }^{W T}$ protein does not affect Lp, but KBTDB13 ${ }^{\text {R408C }}$ increases Lp of actin filaments. (F). Left: relaxation traces of 2 segments of same permeabilized muscle fiber: one incubated with recombinant KBTBD13 ${ }^{W T}$, the other with recombinant KBTBD13 ${ }^{\mathrm{R} 408 \mathrm{C}}$. Right: In healthy control muscle, incubation with KBTBD13 ${ }^{\mathrm{R} 408 \mathrm{C}}$ slows relaxation kinetics. In NEM6 muscle, incubation with KBTBD13 ${ }^{\mathrm{WT}}$ does not affect relaxation kinetics. Student's $t$ tests or Mann-Whitney $U$ tests were performed. For detailed information on number of samples and statistical tests and outcomes, see Supplemental Table 1.

Similar to other proteins that contain repeating Kelch motifs, the resulting KBTBD13 homology model contains a $\beta$ propeller structure. The $\beta$ propeller is located in the $\mathrm{C}$ terminal portion, while the BTB motif adopts a more loosely defined zinc finger in the -terminal region. Intriguingly, protein sequence analysis revealed similarities between KBTBD13 and the actin-binding protein scruin, found in limulus sperm (29). To assess the similarities between the scruin and KBTBD13 $\beta$ propeller motifs, we also developed a homology model for the scruin protein. A different template structure was used for constructing the scruin homology model to avoid bias. We found several similarities between the $\beta$ propeller motif of KBTBD13 and scruin (Figure 6A). In both $\beta$ propeller motifs, 
the majority of cysteines were contained internally within the $\beta$ sheets. In scruin, a single cysteine (Cys837) located in a loop at the surface of the $\beta$ propeller was found to be important for the interaction with filamentous actin (30). Importantly, KBTBD13 has a single cysteine (Cys367) located in a similar position relative to scruin Cys837 (Figure 6A). Scruin is known to bind to filamentous actin, and Cys 837 is important for this binding. These findings suggest that KBTBD13 might interact with actin. Strikingly, the location of 3 KBTBD13 mutations (I369M, K390N and R408C) are in close proximity to Cys367 (Figure 6A).

To test whether KBTDB13 binds to actin, we performed pulldown assays with full-length KBTBD13 and muscle lysates. The pulled down proteins were separated on an SDS-PAGE gel (Figure 6B). One unique band was observed at a height that corresponds to a molecular weight of $40-45 \mathrm{kD}$. The proteins in this band were identified by mass spectrometry. Actin was one of the major proteins identified. Then, to validate whether KBTBD13 also binds to filamentous actin in vitro and whether the most prevalent disease-causing mutation affects this binding, we expressed full-length KBTBD13 and KBTBD13 ${ }^{\mathrm{R} 408 \mathrm{C}}$ and performed a cosedimentation assay with filamentous actin. The data demonstrate that both KBTBD13 and KBTBD13 ${ }^{\mathrm{R} 408 \mathrm{C}}$ bind to filamentous actin with comparable affinities (Figure 6C). As actin-binding properties were not different between KBTBD13 and KBTBD13 ${ }^{\mathrm{R} 408 \mathrm{C}}$, we fitted a single curve using the data from both proteins (shared fit). The resulting binding curve showed a Kd of $3.25 \pm 19.08 \mu \mathrm{M}$ to actin and a maximum binding of approximately $1.08 \pm 1.92 \mu \mathrm{M}$ KBTBD13 to $8 \mu \mathrm{M}$ actin, suggesting a stoichiometry of approximately 1 KBTBD13 molecule to 8 actin molecules. Recombinant KBTBD13 also showed some sedimentation in the absence of actin (Figure 6C, $\mathrm{P}-$ ); however, in the presence of actin $(\mathrm{P}+)$, greater amounts of KBTBD13 were consistently detected in the pellet and all data points are corrected for the amount of KBTBD13 sedimenting in the absence of actin at each concentration. Glutathione S-transferase (GST) alone did not sediment in the presence or absence of actin. Thus, our findings suggest that KBTBD13 is an actin-binding protein.

We next determined the localization of KBTBD13 in muscle fibers. We were unable to detect KBTBD13 using antibodies (we tested commercially available antibodies and 2 custom-made antibodies). Therefore, we generated transgenic Kbtbd13-EGFP and $K b t b d 13^{\mathrm{R} 408 \mathrm{C}}$-EGFP zebrafish. Note that in these fish, the fibers display mosaic expression of KBTBD13; hence, only few cells show labeling. Confocal microscopy analysis suggests that both KBTBD13 and KBTBD13 ${ }^{\mathrm{R} 408 \mathrm{C}}$ localize to the myofibrils, and the fluorescence pattern, throughout the sarcomere with a peak at the Z-disc, appears consistent, with colocalization with actin (Supplemental Figure 8, A and B). Then, to more accurately localize KBTDB13, we made use of APEX - a peroxidase enzyme that can be used to stain samples for EM. The peroxidase was coupled to a GFP-binding peptide that then allowed for EM localization of GFPtagged Kbtbd13. We made a transgenic line of fish that contains the GFP-binding APEX protein and injected these with Kbtbd13-EGFP. As shown in Figure 6D, the EM-APEX data indicate that KBTBD13 localizes to the myofibrils. Within the myofibrils, the A-band (mainly myosin-based thick filaments) and the I-band (mainly actin-based thin filaments) showed dark labeling, indicating that
KBTBD13 binds to the thin filament and the thick filament. Furthermore, the z-discs, where thin filaments from adjacent sarcomeres overlap, show strong labeling. Note that to increase the contrast between KBTBD13-positive and -negative areas, the "normal" EM image (Figure 6D) was converted from gray scale to spectrum scale (Figure 6D), with KBTBD13 in light blue. The EM images of transgenic Kbtbd13 ${ }^{408 C}$-EGFP fish showed localization comparable to that of the transgenic Kbtbd13 ${ }^{\text {WT}}$-EGFP fish (Supplemental Figure $8 \mathrm{C}$ ). Thus, the data from these experiments support the confocal, pull-down, and cosedimentation data showing that KBTBD13 binds to actin in the thin filaments of muscle.

\section{KBTBD13 ${ }^{\mathrm{R} 408 \mathrm{C}}$ increases the flexural rigidity of actin filaments}

To study whether binding of KBTBD13 directly affects the structure of thin filaments, we tested the effect of KBTBD13 ${ }^{\mathrm{WT}}$ and KBTBD $13^{\mathrm{R} 408 \mathrm{C}}$ on the flexural rigidity (assessed by determining the persistence length) of actin filaments in solution. Figure $6 \mathrm{E}$ shows a typical image of actin filaments in solution (Supplemental Videos 4-6 show actin filaments in solution). The results show that KBTBD13 ${ }^{\mathrm{WT}}$ does not change the persistence length of actin filaments compared with that of actin filaments not exposed to $\mathrm{KBTBD}^{\mathrm{WT}}$ (Figure 6E). However, KBTBD13 ${ }^{\mathrm{R} 408 \mathrm{C}}$ significantly increased the persistence length of actin filaments (Figure $6 \mathrm{E})$. These findings show that binding of KBTBD13 ${ }^{\mathrm{R} 408 \mathrm{C}}$ directly increases the stiffness of actin filaments.

\section{KBTBD13 ${ }^{\mathrm{R} 408 \mathrm{C}}$ slows relaxation of human muscle fibers}

To study whether slower relaxation kinetics in NEM6 muscle fibers are a direct effect of mutant KBTBD13, we incubated permeabilized quadriceps single-muscle fibers from control subjects and NEM6 patients with either KBTBD13 ${ }^{\mathrm{WT}}$ or KBTBD13 ${ }^{\mathrm{R} 408 \mathrm{C}}$ and studied contractile function (Figure 6F). In fibers from NEM6 patients, the maximal forces were comparable between groups $\left(108 \pm 20 \mathrm{mN} / \mathrm{mm}^{2}\right.$ for fibers incubated with KBTBD13 ${ }^{\mathrm{WT}}$ and 120 $\pm 17 \mathrm{mN} / \mathrm{mm}^{2}$ for fibers incubated with $\left.\mathrm{KBTBD} 13^{\mathrm{R} 408 \mathrm{C}}\right)$. Incubation with KBTBD13 ${ }^{\mathrm{WT}}$ slightly increased the relaxation kinetics compared with incubation with KBTBD13 ${ }^{\mathrm{R} 408 \mathrm{C}}$ (Figure 6F); however, this difference did not reach significance. In fibers from control subjects, maximal forces were also comparable between groups $\left(125 \pm 9 \mathrm{mN} / \mathrm{mm}^{2}\right.$ for fibers incubated with KBTBD13 ${ }^{\mathrm{WT}}$ and 114 $\pm 9 \mathrm{mN} / \mathrm{mm}^{2}$ for fibers incubated with $\mathrm{KBTBD} 13^{\mathrm{R} 408 \mathrm{C}}$ ). However, incubation with $\mathrm{KBTBD} 13^{\mathrm{R} 408 \mathrm{C}}$ significantly slowed relaxation kinetics compared with incubation with KBTBD13 ${ }^{\mathrm{WT}}$ (Figure 6F).

Together, these findings suggest that in the NEM6 fibers, the KBTBD13 ${ }^{\mathrm{R} 408 \mathrm{C}}$ protein was not replaced by $\mathrm{KBTBD} 13^{\mathrm{WT}}$ protein, but the KBTBD13 ${ }^{\mathrm{WT}}$ protein was added to the endogenous KBTBD13 ${ }^{\mathrm{R} 408 \mathrm{C}}$ protein. Apparently, the amount of endogenous KBTBD13 ${ }^{\mathrm{R} 408 \mathrm{C}}$ protein in the NEM6 fibers was sufficient to maintain the slow-relaxation kinetics. Similarly, in the healthy fibers, KBTBD13 ${ }^{\mathrm{WT}}$ protein was likely not replaced by KBTBD13 ${ }^{\mathrm{R} 408 \mathrm{C}}$ protein, but the KBTBD13 ${ }^{\mathrm{R} 408 \mathrm{C}}$ protein was added to the endogenous KBTBD13 ${ }^{\mathrm{WT}}$ protein. This addition was sufficient to exert an effect on sarcomere relaxation kinetics. These findings are in line with the R408C mutation being a dominant mutation.

Finally, it is important to note that in the experiments with permeabilized muscle fibers, the cytosol is replaced by experimental solutions. Therefore, our findings suggest that KBTBD13 ${ }^{\mathrm{R} 408 \mathrm{C}}$ is 


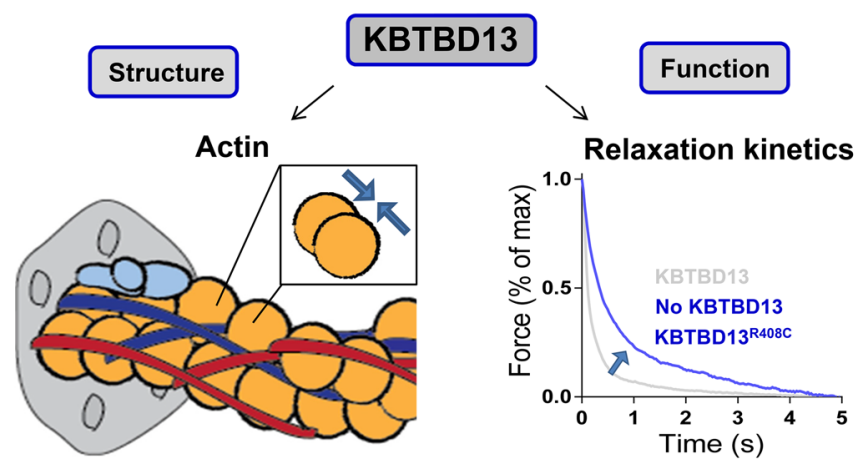

Figure 7. Graphic summary of the proposed pathomechanism in NEM6. This study identified KBTBD13 as an actin-binding protein. Mutations in KBTBD13 alter the structure of the thin filament, thereby impairing muscle relaxation kinetics.

unlikely to act through ubiquitination or disruption of filament assembly/turnover, but rather that it regulates relaxation kinetics by direct binding to sarcomeric structures.

\section{Discussion}

With the use of TMS, we established that the origin of impaired muscle-relaxation kinetics in NEM6 myopathy is myogenic. The pathomechanism underlying the observed impaired muscle-relaxation kinetics was studied using contractility assays in permeabilized muscle fibers and myofibrils isolated from patient biopsies. We discovered that impaired muscle relaxation is sarcomere based. By applying a combination of low-angle x-ray diffraction, superresolution microscopy, modeling of muscle kinetics, and proteomics with genetically modified mouse models and zebrafish, we showed that KBTBD13, the protein affected in NEM6, is an actin-binding protein. Mutations in KBTBD13 slowed relaxation kinetics of muscle through direct, structural effects on the actin-based thin filament. We propose that this pathomechanism is central to NEM6 pathology.

Impaired muscle relaxation in NEM6 is sarcomere based. In vivo muscle-relaxation properties were considerably slower in NEM6 patients compared with healthy controls. TMS has been used before to measure physiological muscle-relaxation kinetics (25, 31-35), but this is the first time, to our knowledge, that this technique has been used to assess muscle relaxation in myopathy. TMS eliminates all influences of the nervous system on muscle relaxation. Consequently, our findings demonstrate that the impaired relaxation kinetics in NEM6 patients are myogenic in nature. With a doubling of relaxation time, the muscle slowness was severe, with profound effects on mobility, as shown in the Supplemental Videos. The NEM6 phenotype, i.e., impaired muscle-relaxation kinetics and the absence of severe weakness (Table 1), distinguishes NEM6 from other nemaline myopathies.

The kinetics of muscle relaxation depend on the kinetics of $\mathrm{Ca}^{2+}$ reuptake by the sarcoplasmic reticulum and the kinetics of thick-filament detachment from the thin filament. Activity levels of phospholamban, a natural inhibitor of the $\mathrm{Ca}^{2+}$ pumps in the sarcoplasmic reticulum, were increased in NEM6 muscle biopsies (Supplemental Figure 1F), suggesting that slowed $\mathrm{Ca}^{2+}$ reuptake might contribute to the slow-relaxation kinetics in muscle of
NEM6 patients. In addition, the findings from histology assays suggest that the relative increase in the area of slow-twitch muscle fibers might contribute as well (slow-twitch fibers have slower contractile kinetics than fast-twitch fibers). However, slow-twitch fiber predominance is a common feature in all forms of NEM and in congenital myopathies in general, whereas clinically, impaired muscle relaxation is specific for NEM6, indicating that the increased area of slow-twitch muscle fibers is unlikely to account for the NEM6 phenotype. The findings from contractility assays in fibers and myofibrils isolated from patient biopsies, assays in which confounding effects of $\mathrm{Ca}^{2+}$ handling by the sarcoplasmic reticulum are absent, revealed that changes in the kinetics of thick-thin-filament detachment are an important contributor to the impaired relaxation kinetics in NEM6 muscle. These changes were present in both slow- and fast-twitch fibers. Thus, impaired sarcomeric relaxation kinetics are a major contributor to slowed muscle-relaxation kinetics in NEM6, irrespective of muscle fiber type.

Thin-filament structure is altered in NEM6 muscle. Low-angle $\mathrm{x}$-ray diffraction studies on muscle fibers revealed that the cause of slower thick-thin filament detachment kinetics might involve structural changes of thin filaments in NEM6 muscle. The position of ALL6, reflecting the left-handed pitch of the thin-filament helix (Figure 4D), was reduced in NEM6 muscle fibers from approximately 5.88 to approximately $5.85 \mathrm{~nm}$, indicating that thin filaments are more tightly wound. This compression $(\sim 0.5 \%$, which translates to $\sim \mathrm{nm}$ on a thin-filament length of $1300 \mathrm{~nm}$ and is therefore not detectable with STED microscopy, Supplemental Figure 2F) might affect the stiffness of thin filaments. The low-angle x-ray diffraction experiments in the newly generated Kbtbd13 ${ }^{R 408 C}$-KI mouse model provided conclusive evidence that thin-filament stiffness is increased in muscle with KBTBD13 ${ }^{\mathrm{R} 408 \mathrm{C}}$.

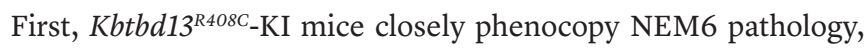
including nemaline bodies in muscle fibers and slow kinetics of muscle relaxation (Figure 5). Second, the simultaneous assessment of force generation and actin subunit spacing (the $2.7 \mathrm{~nm}$ reflection) during low-angle x-ray diffraction experiments in the KI mice revealed that the stiffness of the thin filament was increased from $37 \mathrm{pN}^{*} \mathrm{~nm}^{-1 *} \mu \mathrm{m}^{-1}$ in WT muscles to $49 \mathrm{pN} \times \mathrm{nm}^{-1} \times \mu \mathrm{m}^{-1}$ in KI muscles (Figure $5 \mathrm{M}$ ). The value for WT muscle is comparable to that recently reported for murine $\mathrm{m}$. soleus $\left(30 \mathrm{pN} \times \mathrm{nm}^{-1} \times\right.$ $\mu \mathrm{m}^{-1} ;$ ref. 27). On the other hand, the value for KI muscle is likely an underestimation, as in the determination of myofibrillar area (required to calculate the number of individual thin filaments), we did not take into account the area occupied by nemaline rods. Figure 5, C and D, suggests that this area is significant. Thus, the actual stiffness of thin filaments in KI muscle might even be considerably higher than the value here reported.

To evaluate whether the increased thin-filament stiffness slows muscle-relaxation kinetics, we turned to simulations of sarcomere contractility kinetics, using the model of paired compliant thin and thick filaments described by Campbell (28) (Supplemental Figure 7). In these simulations, the rate of relaxation reflects the speed at which the thick-filament-based myosin heads detach from actin-binding sites on the thin filament. This detachment rate is accelerated by allowing myosin heads to move relative to actin. In real muscles, the relative movement between the myosin heads and actin-binding sites results from, among others, 
thick- and thin-filament compliance (inverse of stiffness). In brief, during sarcomeric relaxation, the $\mathrm{Ca}^{2+}$ concentration near the sarcomeres falls. If a myosin head detaches under these conditions, the binding site on actin has a high probability of deactivating before another myosin head can bind. Thus, myosin heads that are detached by movement of thick filaments relative to thin filaments (due to thin and thick-filament compliance, i.e., the reciprocal of stiffness) are not replaced. The prevailing force must thus be borne by a smaller number of myosin head-actin interactions. This in turn stretches the remaining interactions and accelerates their detachment. The net result is a positive feedback loop that causes thin-thick-filament detachment. Our simulation results suggest that increasing thin-filament stiffness decreases the positive feedback and slows the rate of relaxation. To our knowledge, this is the first study that links increased thin-filament stiffness with slow sarcomeric relaxation kinetics. The findings obtained provide insights into the mechanisms underlying the previously reported fast relaxation kinetics of myofibrils from nebulin-deficient mice (36): in the absence of nebulin, the thin filaments were more compliant (27).

It should be noted that the magnitude of thin-filament stiffening in NEM6 muscle is unknown, and therefore we modeled the effect of very stiff thin filaments. Hence, we cannot rule out that in NEM6 muscle, the effect of thin-filament stiffness on relaxation kinetics is less pronounced than the model predicts. Furthermore, in addition to thin-filament stiffness, increased thin-filament activation in NEM6 muscle might contribute to the slow-relaxation kinetics. Interestingly, increased thin-filament activation would not only slow-relaxation kinetics, but would also increase the calcium sensitivity of force, as indeed observed in NEM6 fibers (Supplemental Figure 2, D and E) and indirectly in muscle of $K b t b d 13^{R 408 C}-\mathrm{KI}$ mice (Supplemental Figure $5 \mathrm{H}$ ). We speculate that the KBTBD13 ${ }^{\mathrm{R} 408 \mathrm{C}}$-induced increased flexural rigidity of thin filaments might play a role (Figure 6E; 30\% increase in the persistence length of actin filaments exposed to recombinant KBTB$\mathrm{D} 13^{\mathrm{R} 408 \mathrm{C}}$ protein). This speculation is based on previous reports indicating that, similarly to $\mathrm{KBTBD} 13^{\mathrm{R} 408 \mathrm{C}}$, the actin-binding protein phalloidin (a) increases the persistence length of actin filaments (37) with (b) a concomitant increase of thin-filament activation (38). Finally, the KBTBD13-based effects on thin-filament activation and on thin-filament stiffness might act in concert to modulate muscle-relaxation kinetics in NEM6 muscle, a possibility that warrants further investigation.

\section{KBTBD13 modulates thin-filament function}

Our data indicate that the increased stiffness of thin filaments in NEM6 muscle is a direct consequence of binding of mutant KBTBD13 to actin. KBTBD13 is a muscle-specific protein composed of an N-terminal BTB domain and a C-terminal Kelchrepeat domain. The role of KBTBD13 in muscle structure and function has been largely unknown. Previous work suggested that KBTBD13 is a substrate adaptor for Cullin-3, a muscle-specific ubiquitin ligase, and thereby implicated the ubiquitin/proteasome pathway in the pathogenesis of KBTBD13-associated $\operatorname{NEM}(24,39)$. The present study reveals that KBTBD13 has additional functions, namely that of an actin-binding protein that modulates the relaxation kinetics of muscle. Homology model- ing suggested that KBTBD13 binds to actin (Figure 6A), which was confirmed by pull-down assays and cosedimentation assay with filamentous actin (Figure 6, B and C). APEX-based EM of GFP-tagged mutant and WT KBTBD13 in zebrafish showed localization consistent with KBTBD13 binding to actin in intact muscle fibers (Figure 6D). Of interest, the binding assay predicted a 1:8 stoichiometry of KBTBD13 to actin. This is similar to the stoichiometry of the regulatory proteins tropomyosin and troponin to actin (both 1:7). Tropomyosin, troponin, and 7 actin monomers together form one regulatory unit, and a serial sequence of these regulatory units control thin-filament activation and deactivation. This raises the intriguing possibility of KBTBD13 acting in concert with actin, tropomyosin, and troponin to fine-tune the kinetics of muscle contractility. It should be noted that the APEX-based EM studies in zebrafish suggested that KBTBD13 also localizes to the cytoplasm and the thick filament (Figure 6D). Indeed, the low-angle x-ray diffraction studies in Kbtbd13KI mice showed reduced thick-filament backbone periodicities (M6 and $2.8 \mathrm{~nm}$ reflections; Supplemental Figure 5, K and L), suggesting an altered structure. Thick-filament stiffness, however, was unaffected, as indicated by the unaltered $\Delta 2.8 \mathrm{~nm}$ reflection during muscle activation (Supplemental Figure $5 \mathrm{M}$ ). The functional significance of KBTBD13 localization to the thick filament is unclear and warrants further investigation. The cytoplasm localization is in agreement with previous work from Sambuughin et al. in so-called "premyofibrils" of c2c12 cells, which suggested cytosolic localization, but no myofibrillar localization (20). Perhaps localization of KBTBD13 depends on the developmental stage of muscle and KBTBD13 doesn't localize to the actin filament until after the premyofibril stage.

To date, 3 disease-causing mutations in KBTBD13 have been described, of which the p.R408C mutation is the most frequently described one. Here, we report 2 mutations in KBTBD13: p.I369M and p.E83Q. Notably, I369 is located near K390, R408, and the cysteine that might be responsible for actin binding (C367, Figure 6A). This observation, in combination with the observed structural and functional changes in the actin-based thin filament in muscle fibers from NEM6 patients and Kbtbd13 ${ }^{R 408 C}$-KI mice, supports our hypothesis that KBTBD13 mutations affect relaxation kinetics through direct effects on the thin filament. The majority of patients included in the present study have the KBTBD $13^{R 408 C}$ mutation (Table 1). For this reason, we generated recombinant KBTB$\mathrm{D} 13^{\mathrm{R} 408 \mathrm{C}}$ and observed that its binding affinity for filamentous actin was comparable to that of KBTBD13 ${ }^{\mathrm{WT}}$, despite the mutation being close to $\mathrm{C} 367$ (Figure 6A). This suggests that the mechanism underlying the observed slowing of relaxation kinetics of human muscle fibers exposed to KBTBD13 ${ }^{\mathrm{R} 408 \mathrm{C}}$ (Figure 6F) does not involve reduced or increased binding to actin of mutant KBTBD13, but rather an effect on the structure of the thin filament once bound to actin. The nature of this effect is unknown. We propose that $K B T B D 13^{R 408 C}$ is a gain-of-function mutation. This proposition

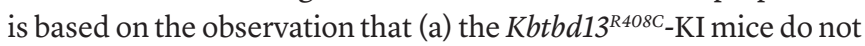
phenocopy the mice that are deficient in KBTBD13 (e.g., unlike the $K b t b d 13-\mathrm{KO}$ mice, the $K b t b d 13^{R 408 C}$ mice have slower kinetics of muscle relaxation; Figure $5 \mathrm{H}$ ); and (b) KBTBD13 ${ }^{\mathrm{WT}}$ protein did not affect the flexural rigidity of thin filaments, whereas KBTBD13 ${ }^{\mathrm{R} 408 \mathrm{C}}$ increased the rigidity (Figure 6E). Thus, KBTBD $13^{\mathrm{WT}}$ protein does 
not affect thin-filament structure and muscle-relaxation kinetics, whereas KBTBD13 ${ }^{\mathrm{R} 408 \mathrm{C}}$ slows muscle-relaxation kinetics by direct effects on thin-filament structure.

How the putative role of KBTBD13 in ubiquitin-ligase activity is involved in these effects on muscle-relaxation kinetics is unknown. An important aspect of the experimental approach was that in the assays in which the effect of KBTBD13 on relaxation was studied, permeabilized muscle fibers were used (Figure $6 \mathrm{~F}$ ). In permeabilized muscle fibers, the cytosol is replaced by an experimental solution in which no ubiquitin and conjugation enzymes are present. Thus, in this context, the modulation of relaxation kinetics by $\mathrm{KBTBD} 13^{\mathrm{R} 408 \mathrm{C}}$ protein (Figure $6 \mathrm{~F}$ ) must be a consequence of direct binding to sarcomeric proteins, presumably actin, rather than of ubiquitin ligase or other cytosolic activity. Note that the newly identified mutation E83Q is located in the BTB domain of KBTBD13, whereas the other 4 mutations are located in the Kelch domain. The BTB domain interacts with $\mathrm{Cul3}$, whereas the Kelch domain provides an adaptor function for substrate recognition $(24,39)$. Based on our findings, we postulate that actin is targeted by KBTBD13's Kelch domain, and we speculate that the mutation in the BTB domain may affect sarcomere function through changes in ubiquitination.

In conclusion, this study identified KBTBD13 as an actin-binding protein, a property that when affected by mutations alters the structure of the thin filament, thereby impairing muscle-relaxation kinetics (Figure 7). We propose that this pathomechanism is central to the NEM6 phenotype.

\section{Methods}

Please see the Supplemental Methods for a detailed description of TMS, muscle biopsies, permeabilized muscle-fiber mechanics, myosin heavy chain isoform composition determination, myofibril mechanics, recombinant KBTBD13 and KBTBD13 ${ }^{\mathrm{R} 408 \mathrm{C}}$ protein expression, actin and KBTBD13/KBTBD13 ${ }^{\mathrm{R} 408 \mathrm{C}}$ cosedimentation assay, pulldown assay with recombinant KBTBD13 proteins, mass spectrometry, KBTBD13 and KBTBD13 ${ }^{\mathrm{R} 408 \mathrm{C}}$ incubation assay in human muscle fibers, generation of the Kbtbd13-KO and Kbtbd13 ${ }^{\mathrm{R} 408 \mathrm{C}}-\mathrm{KI}$ mouse models, intact muscle mechanics, homology modeling, KBTBD13 localization in zebrafish, antibodies used for Western blot, flexural rigidity of actin filaments, EM, immunohistochemistry, $\mathrm{Ca}^{2+}$ reuptake, low-angle $\mathrm{x}$-ray diffraction, and stimulated emission-depletion microscopy.

Statistics. Data are presented as mean \pm SEM or as median with interquartile range (normally distributed, not normally distributed, respectively). For statistical analyses, 2-tailed $t$ tests or Mann-Whitney $U$ tests were used. A $P$ value of less than 0.05 was considered statistically significant. For detailed information on the number of samples and statistical tests and outcomes, please see Supplemental Table 1.

Study approval. Experimental procedures for human studies were approved by the institutional ethics committee and performed in accordance with ethical standards laid down in the Declaration of Helsinki. All biopsies were collected following informed consent supervised by the Radboud University Institutional Review Board. All murine experiments were approved and conducted under the supervision of the University of Arizona IACUC and followed the NIH guidelines Using Animals in Intramural Research.

\section{Author contributions}

JMDW, CACO, DER, BB, RAB, RJBR, and TI designed research studies. JMDW, JPM, MY, RVDP, SS, SC, MVDL, MW, SJPB, ESBVK, SL, MP, TES, AAR, VO, GR, TEH, ZX, CNJ, FL, B Kiss, NLV, RAB, MM, LN, RJR, B Küsters, JD, KC, WM, and EM conducted experiments. JMDW, JPM, MY, RVDP, SS, SC, MVDL, MW, SJPB, ESBVK, SL, MP, TES, AAR, VO, GR, TEH, ZX, CNJ, FL, B Kiss, NLV, RAB, MM, LN, RJR, B Küsters, JD, KC, WM, and EM acquired data. JMDW, JPM, MY, RVDP, SS, SC, MVDL, MW, SJPB, ESBVK, SL, MP, TES, AAR, VO, GR, TEH, ZX, CNJ, FL, B Kiss, NLV, RAB, MM, LN, RJR, B Küsters, JD, KC, WM, NBR, and EM analyzed data. JMDW, JPM, CACO, NCV, BGMVE, HG, AHB, and NR wrote the manuscript. Both JMDW and JPM provided the majority of work in this study: JMDW took responsibility for the preclinical part and JPM for the clinical work. As during the study progress, the preclinical part increased significantly over the clinical work, JMDW is now listed as first author and JPM as second.

\section{Acknowledgments}

This work was supported by the Dutch Foundation for Scientific Research (VIDI 016.126.319 to CACO); the Princess Beatrix Muscle Foundation (W.OR17-08 to CACO, NCV, and BGMVE); H2020-MSCA-RISE-2014 (645648 Muscle Stress Relief to CACO); the Advanced Photon Source (DE-AC02-06CH11357); A Foundation Building Strength for Nemaline Myopathy (to CACO); the National Health and Medical Research Council (NHMRC) Early Career Fellowship (APP1121651 to MY); the National Institute of Child Health and Human Development (NIH R01 HD075802 to AHB); the Muscular Dystrophy Association (USA) (MDA602235 to AHB); the National Institute of Arthritis and Musculoskeletal and Skin Diseases (NIH R01 AR053897 to HG), the NIH (HL133359 to KC). This research used resources of the Advanced Photon Source, a U.S. Department of Energy (DOE) Office of Science User Facility operated for the DOE Office of Science by Argonne National Laboratory under contract no. DE-AC02-06CH11357. This project was supported by grant 9 P41 GM103622 from the National Institute of General Medical Sciences of the NIH. Use of the Pilatus 3 1M detector was provided by grant 1S100D018090-01 from the National Institute of General Medical Sciences. The content is solely the responsibility of the authors and does not necessarily reflect the official views of the National Institute of General Medical Sciences or the NIH. MP was funded by a postdoctoral grant from the Swedish Research Council (2015-00385).

Address correspondence to: Coen Ottenheijm, Amsterdam University Medical Center, Department of Physiology, $\mathrm{O} \mid 2$ building, De Boelelaan 1118, 1081 HV Amsterdam, Netherlands. Phone: 31.0.6.27339910; Email: c.ottenheijm@vumc.nl.
1. Poggesi C, Tesi C, Stehle R. Sarcomeric determinants of striated muscle relaxation kinetics. Pflugers Arch. 2005;449(6):505-517.

2. Regnier M, Cheng Y. Finally, we can relax: a new generation of muscle models that incorporate sarcomere compliance. Biophys J. 2016;110(3):521-522.

3. Kortman HG, Veldink JH, Drost G. Positive muscle phenomena--diagnosis, pathogenesis and associated disorders. Nat Rev Neurol. 2012;8(2):97-107.

4. Gommans IM, et al. A new phenotype of autoso- 
mal dominant nemaline myopathy. Neuromuscul Disord. 2002;12(1):13-18.

5. Gommans IM, et al. A locus on chromosome $15 \mathrm{q}$ for a dominantly inherited nemaline myopathy with core-like lesions. Brain. 2003;126(Pt 7):1545-1551.

6. Pauw-Gommans IM, Gerrits KH, de Haan A, van Engelen BG. Muscle slowness in a family with nemaline myopathy. Neuromuscul Disord. 2006;16(8):477-480.

7. Colombo I, et al. Congenital myopathies: Natural history of a large pediatric cohort. Neurology. 2015;84(1):28-35.

8. Ilkovski B, et al. Nemaline myopathy caused by mutations in the muscle alpha-skeletal-actin gene. Am J Hum Genet. 2001;68(6):1333-1343.

9. Laing NG, et al. A mutation in the alpha tropomyosin gene TPM3 associated with autosomal dominant nemaline myopathy. Nat Genet. 1995;9(1):75-79.

10. Donner K, et al. Mutations in the beta-tropomyo$\sin$ (TPM2) gene--a rare cause of nemaline myopathy. Neuromuscul Disord. 2002;12(2):151-158.

11. Pelin K, et al. Mutations in the nebulin gene associated with autosomal recessive nemaline myopathy. Proc Natl Acad Sci USA. 1999;96(5):2305-2310.

12. Yuen M, et al. Leiomodin-3 dysfunction results in thin filament disorganization and nemaline myopathy. J Clin Invest. 2014;124(11):4693-4708.

13. Johnston JJ, et al. A novel nemaline myopathy in the Amish caused by a mutation in troponin $\mathrm{T} 1$. Am J Hum Genet. 2000;67(4):814-821.

14. Sandaradura SA, et al. Nemaline myopathy and distal arthrogryposis associated with an autosomal recessive TNNT3 splice variant. Hum Mutat. 2018;39(3):383-388.

15. Agrawal PB, et al. Nemaline myopathy with minicores caused by mutation of the CFL2 gene encoding the skeletal muscle actin-binding protein, cofilin-2. Am JHum Genet. 2007;80(1):162-167.

16. Malfatti E, Böhm J, Lacène E, Beuvin M, Romero NB, Laporte J. A premature stop codon in MYO18B is associated with severe nemaline myopathy with cardiomyopathy. J Neuromuscul Dis. 2015;2(3):219-227.

17. Miyatake S, et al. Biallelic mutations in MYPN, encoding myopalladin, are associated with childhood-onset, slowly progressive nemaline myopathy. Am J Hum Genet. 2017;100(1):169-178.

18. Ravenscroft G, et al. Mutations in KLHL4O are a frequent cause of severe autosomal-recessive nemaline myopathy. Am J Hum Genet. 2013;93(1):6-18.

19. Gupta VA, et al. Identification of KLHL41 mutations implicates BTB-Kelch-mediated ubiquitination as an alternate pathway to myofibrillar disruption in nemaline myopathy. Am J Hum Genet. 2013;93(6):1108-1117.

20. Sambuughin N, et al. Dominant mutations in KBTBD13, a member of the BTB/Kelch family, cause nemaline myopathy with cores. Am J Hum Genet. 2010;87(6):842-847.

21. de Winter JM, Ottenheijm CAC. Sarcomere dysfunction in nemaline myopathy. J Neuromuscul Dis. 2017;4(2):99-113.

22. Ottenheijm CA, Witt CC, Stienen GJ, Labeit S, Beggs AH, Granzier H. Thin filament length dysregulation contributes to muscle weakness in nemaline myopathy patients with nebulin deficiency. Hum Mol Genet. 2009;18(13):2359-2369.

23. Winter JM, et al. Mutation-specific effects on thin filament length in thin filament myopathy. Ann Neurol. 2016;79(6):959-969.

24. Sambuughin N, et al. KBTBD13 interacts with Cullin 3 to form a functional ubiquitin ligase. Biochem Biophys Res Commun. 2012;421(4):743-749.

25. Molenaar JP, Voermans NC, de Jong LA, Stegeman DF, Doorduin J, van Engelen BG. Repeatability and reliability of muscle relaxation properties induced by motor cortical stimulation. J Appl Physiol. 2018;124(6):1597-1604.

26. Millman BM. The filament lattice of striated muscle. Physiol Rev. 1998;78(2):359-391.

27. Kiss B, et al. Nebulin stiffens the thin filament and augments cross-bridge interaction in skeletal muscle. Proc Natl Acad Sci USA. 2018;115(41):10369-10374.

28. Campbell KS. Compliance accelerates relaxation in muscle by allowing myosin heads to move relative to actin. Biophys J. 2016;110(3):661-668.

29. Schmid MF, Agris JM, Jakana J, Matsudaira P,
Chiu W. Three-dimensional structure of a single filament in the Limulus acrosomal bundle: scruin binds to homologous helix-loop-beta motifs in actin. J Cell Biol. 1994;124(3):341-350.

30. Sun S, Footer M, Matsudaira P. Modification of Cys-837 identifies an actin-binding site in the beta-propeller protein scruin. Mol Biol Cell. 1997;8(3):421-430.

31. Molenaar JP, McNeil CJ, Bredius MS, Gandevia SC. Effects of aging and sex on voluntary activation and peak relaxation rate of human elbow flexors studied with motor cortical stimulation. Age (Dordr). 2013;35(4):1327-1337.

32. Hunter SK, Butler JE, Todd G, Gandevia SC, Taylor JL. Supraspinal fatigue does not explain the sex difference in muscle fatigue of maximal contractions. J Appl Physiol. 2006;101(4):1036-1044.

33. Hunter SK, Todd G, Butler JE, Gandevia SC, Taylor JL. Recovery from supraspinal fatigue is slowed in old adults after fatiguing maximal isometric contractions. J Appl Physiol. 2008;105(4):1199-1209.

34. McNeil CJ, Butler JE, Taylor JL, Gandevia SC. Testing the excitability of human motoneurons. Front Hum Neurosci. 2013;7:152.

35. Todd G, Taylor JL, Butler JE, Martin PG, Gorman $\mathrm{RB}, \mathrm{Gandevia} S \mathrm{SC}$. Use of motor cortex stimulation to measure simultaneously the changes in dynamic muscle properties and voluntary activation in human muscles. JAppl Physiol. 2007;102(5):1756-1766.

36. Ottenheijm CA, et al. Deleting exon 55 from the nebulin gene induces severe muscle weakness in a mouse model for nemaline myopathy. Brain. 2013;136(Pt 6):1718-1731.

37. Pfaendtner J, Lyman E, Pollard TD, Voth GA. Structure and dynamics of the actin filament. JMol Biol. 2010;396(2):252-263.

38. Bukatina AE, Fuchs F, Brandt PW. Thin filament activation by phalloidin in skinned cardiac muscle. J Mol Cell Cardiol. 1995;27(6):1311-1315.

39. Gupta VA, Beggs AH. Kelch proteins: emerging roles in skeletal muscle development and diseases. Skelet Muscle. 2014;4:11. 